\title{
Penetration, Radiation and Scattering for a Cavity-backed Gap in a Corner
}

\author{
Danilo Erricolo, Senior Member, IEEE, Piergiorgio L. E. Uslenghi, Fellow, IEEE
}

\begin{abstract}
A partially covered cavity, or trench, located along the edge of two intersecting metallic walls perpendicular to each other is considered. The cross section of the cavity is a quarter ellipse, and is slotted along the interfocal distance. The cavity is filled with a material isorefractive to that occupying the quarter-space between the walls. This two-dimensional boundary-value problem is solved exactly, when the primary field is either a plane wave of arbitrary polarization and direction of incidence, or an electric or magnetic line source. Numerical results are given and discussed.
\end{abstract}

Keywords - Electromagnetic radiation, electromagnetic scattering, complex media, cavities, isorefractive media, Mathieu functions.

\section{INTRODUCTION}

A PARTIALLY covered trench located at the corner of two metallic walls perpendicular to each other is considered. The cross section of the trench is a quarter ellipse, and it is slotted from the focus to the center of the ellipse; the trench is partially covered by a thin metal strip extending outward from the focal line, as part of the metal wall under which the trench is flush mounted. The trench is filled with a material that is isorefractive to the medium (e.g. air) filling the quarter space between the walls.

This boundary-value problem is solved exactly in the frequency domain, when the primary source is either a plane wave of arbitrary direction of incidence and polarization, or an electric or magnetic line source parallel to the walls and located either outside or inside the trench. The field components are expanded in infinite series of eigenfunctions that are products of radial and angular Mathieu functions, in the Stratton-Chu notation [1],[2]. The modal coefficients in the expansion of the secondary fields are analytically determined by imposing the boundary conditions. The technique utilized in obtaining this new canonical solution is akin to that used for a slotted semielliptical channel [3], that led to numerical results in excellent agreement with integral equation approaches $[4][5][6]$. Some preliminary results for E-polarized plane wave incidence were reported previously in [7].

Cavity-filled problems arise, for example, when antennas are located inside a cavity at the surface of a platform such as an airplane, a missile or a satellite. The smoothness of the platform surface is dictated by aerodynamical or other considerations, and is achieved by filling the cavity, either

The authors are with the Department of Electrical and Computer Engineering, University of Illinois at Chicago, 851 South Morgan St., Chicago, Illinois 60607, USA. E-mail: derricol@ece.uic.edu, uslenghi@uic.edu. This research was supported by the U.S. Department of Defense under MURI grant F49620-01-1-0436. Additionally, this work was supported in part by a grant of computer time from the DoD High Performance Computing Modernization Program at ASC. totally or partially (as in the case of a radome), with a material that is transparent to electromagnetic radiation. A numerical study of this type of boundary-value problems may encounter difficulties, such as estimating the portion of platform surface surrounding the cavity structure that needs to be taken into account in the numerical modeling, or evaluating fields in the vicinity of geometric singularities.

The canonical solutions presented herein are the only exact, analytical solutions available for a trench located at the corner of two walls. Aside from the intrinsic importance of solving exactly a complicated boundary-value problem involving a cavity, a sharp edge and two different penetrable materials, these canonical solutions constitute a benchmark for the validation of frequency-domain computer codes. The geometry of the problem is illustrated in section II, and the exact solutions for plane wave incidence and line sources are obtained in sections III and IV, respectively. Some numerical results are presented and discussed in Section V. The time-dependence factor $\exp (+j \omega t)$ is omitted throughout.

\section{Geometry of the PROBlem}

A cross-sectional view of the structure in a plane $z=$ constant is shown in Fig. 1.

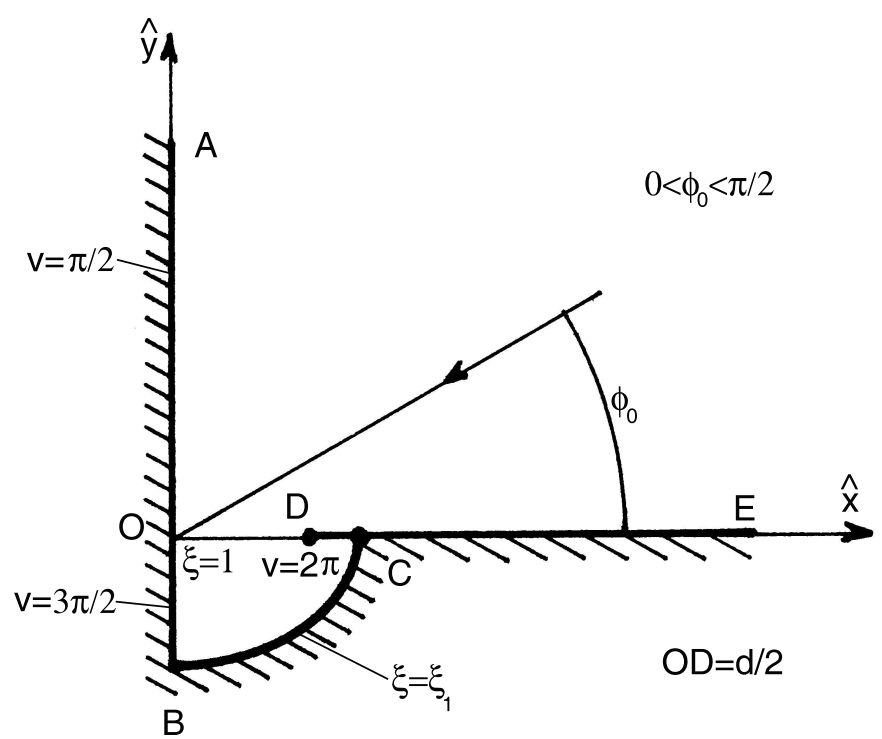

Fig. 1. Geometry of the problem.

The metallic walls $O A(x=0)$ and $O E(y=0)$ are perpendicular to each other. The trench $O B C$ is flushmounted under the horizontal wall $O E$, and its cross section is a quarter ellipse with semi-major axis $O C$ and semi- 
minor axis $O B$. The wall $\mathrm{OE}$ is slotted along the slit of width $O D$ equal to half the interfocal distance $d$ of the elliptical trench. The trench is partially covered by the thin metal baffle $D C$. The rectangular coordinates $(x, y, z)$ are related to the elliptic cylinder coordinates $(u, v, z)$ by:

$$
\begin{aligned}
x & =\frac{d}{2} \cosh u \cos v, \\
y & =\frac{d}{2} \sinh u \sin v, \\
z & =z
\end{aligned}
$$

where $0 \leq u<\infty, 0 \leq v \leq 2 \pi,-\infty<z<\infty$. It is expedient to introduce the coordinates

$$
\xi=\cosh u, \quad \eta=\cos v
$$

with $1 \leq \xi<\infty$ and $-1 \leq \eta \leq 1$. Curves with $\xi$ constant are ellipses and curves with $\eta$ constant are hyperbolas. The half-plane $D E$ corresponds to $v=0$, the surface $D C$ of the baffle inside the trench corresponds to $v=2 \pi$, whereas the portions $O A$ and $O B$ of the vertical wall outside and inside the trench are given by $v=\pi / 2$ and $v=3 \pi / 2$, respectively; finally, the curved surface $B C$ of the trench corresponds to $\xi=\xi_{1}$, and the surface $O D$ of the slit to $\xi=1$ (see Fig. 1). The semi-interfocal distance $O D=d / 2$, the semi-major axis $O C=d \xi_{1} / 2$, and the semi-minor axis $O B=(d / 2) \sqrt{\xi_{1}^{2}-1}$.

The medium filling the quarter space $(x>0, y>0)$ between the walls has an electric permittivity $\varepsilon_{1}$ and a magnetic permeability $\mu_{1}$, whereas the material filling the trench has permittivity $\varepsilon_{2}$ and permeability $\mu_{2}$. The two media are isorefractive, i.e.

$$
\varepsilon_{1} \mu_{1}=\varepsilon_{2} \mu_{2}
$$

so that the propagation constant

$$
k=\omega \sqrt{\varepsilon_{h} \mu_{h}}, \quad(h=1,2)
$$

is the same for both media, whilst the intrinsic impedances

$$
Z_{h}=\sqrt{\mu_{h} / \varepsilon_{h}}, \quad(h=1,2) .
$$

are, in general, different from each other. It is important to understand that condition (5) is necessary for one-to-one mode matching of the fields inside and outside the trench, and the consequent analytical determination of the modal coefficients. If (5) were not satisfied, an analytical solution would be precluded and one would have to deal with the inversion of an infinite matrix for the determination of the modal coefficients. Finally, in the following it is convenient to introduce the dimensionless parameter

$$
c=\frac{k d}{2}=\pi \frac{d}{\lambda} .
$$

where $\lambda$ is the wavelength.

The geometry studied herein and shown in Fig. 1 may be considered a particular case of the slotted channel examined in [3], by inserting a PEC wall in the plane $x=0$. As such, the results obtained in this paper may be derived by combining the formulas in [3] with the method of images, and this is essentially the technique utilized in sections III and IV below. However, the derivations are not trivial; in particular, the following two formulas for the angular Mathieu functions are needed, in addition to all the formulas listed in the Appendix of [3]:

$$
\begin{aligned}
& \left.\frac{\partial}{\partial v} \operatorname{Se}_{2 \mathrm{~m}}(\mathrm{c}, \eta)\right|_{v=\pi / 2, v=3 \pi / 2}=0 \\
& \left.\mathrm{So}_{2 \mathrm{~m}}(\mathrm{c}, \eta)\right|_{v=\pi / 2}=\left.\operatorname{So}_{2 \mathrm{~m}}(\mathrm{c}, \eta)\right|_{v=3 \pi / 2}=0
\end{aligned}
$$

\section{Plane wave incidence}

Any incident plane wave in the quarter space $(x>$ $0, y>0)$ may be considered as a superposition of an Epolarized wave (electric field parallel to the z-axis) and an $\mathrm{H}$-polarized wave (magnetic field parallel to the z-axis). The two polarization cases are treated separately.

\section{A. E-polarization}

Consider an incident plane wave whose direction of propagation forms the angle $\varphi_{0}$ with the negative $x$ axis and the angle $\pi / 2-\varphi_{0}$ with the negative $y$ axis $\left(0<\varphi_{0}<\pi / 2\right.$, see Fig. 1), and whose electric field is given by:

$$
\underline{E}^{i}=\hat{z} E_{z}^{i}=\hat{z} e^{j k\left(x \cos \varphi_{0}+y \sin \varphi_{0}\right)},\left(0<\varphi_{0}<\pi / 2\right),
$$

where $\hat{z}$ is a unit vector in the positive z-direction. The incident field (11) may be expanded in a series of ellipticcylinder wave functions [2]:

$$
\begin{gathered}
E_{z}^{i}=\sqrt{8 \pi} \sum_{m=0}^{\infty}\left[\frac{j^{m}}{N_{m}^{(e)}} \operatorname{Re}_{\mathrm{m}}^{(1)}(\mathrm{c}, \xi) \operatorname{Se}_{\mathrm{m}}(\mathrm{c}, \eta) \operatorname{Se}_{\mathrm{m}}\left(\mathrm{c}, \cos \varphi_{0}\right)\right. \\
\left.+\frac{j^{m}}{N_{m}^{(o)}} \operatorname{Ro}_{\mathrm{m}}^{(1)}(\mathrm{c}, \xi) \operatorname{So}_{\mathrm{m}}(\mathrm{c}, \eta) \operatorname{So}_{\mathrm{m}}\left(\mathrm{c}, \cos \varphi_{0}\right)\right],
\end{gathered}
$$

where $\operatorname{Re}_{\mathrm{m}}^{(1)}$ and $\mathrm{Ro}_{\mathrm{m}}^{(1)}$ are even and odd radial Mathieu functions of the first kind, $\mathrm{Se}_{\mathrm{m}}$ and $\mathrm{So}_{\mathrm{m}}$ are even and odd angular functions, and $N_{m}^{(e),(o)}$ are normalization coefficients [1], [2].

The total electric field in the quarter-space $(x \geq 0, y \geq$ 0 ) may be written as the sum of a geometric-optics field $E_{1 z}^{g o}$ due to the corner reflector without the trench and a diffracted field $E_{1 z}^{d}$ due to the presence of the trench:

$$
E_{1 z}=E_{1 z}^{g o}+E_{1 z}^{d} .
$$

The geometric-optics field is the sum of four terms (see Fig. 2):

$$
E_{1 z}^{g o}=E_{z}^{i}+E_{z}^{O E}+E_{z}^{O A}+E_{z}^{O A, O E}
$$

where the field $E_{z}^{O E}$ corresponds to a wave with incidence angle $2 \pi-\varphi_{0}$ multiplied by a reflection coefficient -1, the field $E_{z}^{O A}$ corresponds to a wave with incidence angle $\pi-\varphi_{0}$ multiplied by a reflection coefficient -1 , and the field $E_{z}^{O A, O E}$ corresponds to a doubly-reflected wave, i.e. a wave with incidence angle $\pi+\varphi_{0}$. When the even and 


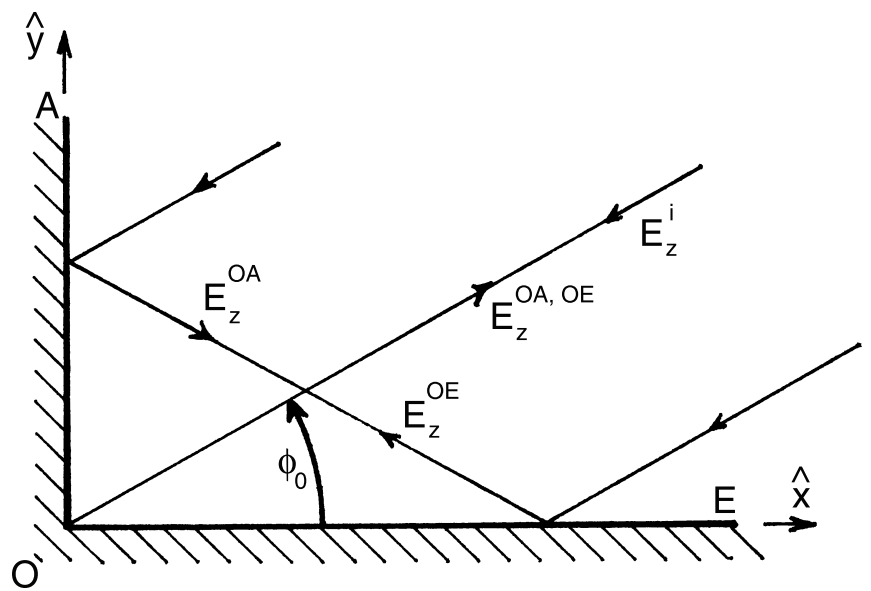

Fig. 2. Geometric-optics contribution for plane-wave incidence.

odd functions of $\varphi_{0}$ are separated out in the various field components, it is found that the overall geometric-optics field is:

$$
E_{1 z}^{g o}=8 \sqrt{2 \pi} \sum_{\ell=0}^{\infty} \frac{(-1)^{\ell}}{N_{2 \ell}^{(o)}} \operatorname{Ro}_{2 \ell}^{(1)}(\mathrm{c}, \xi) \operatorname{So}_{2 \ell}(\mathrm{c}, \eta) \operatorname{So}_{2 \ell}\left(\mathrm{c}, \cos \varphi_{0}\right) .
$$

As a consequence of the boundary conditions on the two walls, only the odd functions of even order remain in the geometric-optics contribution. The diffracted field must satisfy the boundary conditions on the walls and the twodimensional radiation condition, hence it must be of the type:

$E_{1 z}^{d}=8 \sqrt{2 \pi} \sum_{\ell=0}^{\infty} \frac{(-1)^{\ell}}{N_{2 \ell}^{(o)}} a_{\ell} \operatorname{Ro}_{2 \ell}^{(4)}(\mathrm{c}, \xi) \operatorname{So}_{2 \ell}(\mathrm{c}, \eta) \operatorname{So}_{2 \ell}\left(\mathrm{c}, \cos \varphi_{0}\right)$,

where $\mathrm{Ro}_{2 \ell}^{(4)}$ is the radial Mathieu function of the fourth kind, and the modal coefficients $a_{\ell}$ are to be determined.

The total electric field inside the trench may be written as

$$
\begin{aligned}
& E_{2 z}=8 \sqrt{2 \pi} \sum_{\ell=0}^{\infty} \frac{(-1)^{\ell}}{N_{2 \ell}^{(o)}} a_{\ell}\left[\frac{\mathrm{Ro}_{2 \ell}^{(4)}\left(\mathrm{c}, \xi_{1}\right)}{\operatorname{Ro}_{2 \ell}^{(1)}\left(\mathrm{c}, \xi_{1}\right)} \mathrm{Ro}_{2 \ell}^{(1)}(\mathrm{c}, \xi)-\right. \\
&\left.\operatorname{Ro}_{2 \ell}^{(4)}(\mathrm{c}, \xi)\right] \operatorname{So}_{2 \ell}(\mathrm{c}, \eta) \operatorname{So}_{2 \ell}\left(\mathrm{c}, \cos \varphi_{0}\right),
\end{aligned}
$$

where

$$
\begin{aligned}
& a_{\ell}= \\
& \frac{\operatorname{Ro}_{2 \ell}^{(1)^{\prime}}(\mathrm{c}, 1) \mathrm{Ro}_{2 \ell}^{(1)}\left(\mathrm{c}, \xi_{1}\right)}{\zeta \operatorname{Ro}_{2 \ell}^{(1)^{\prime}}(\mathrm{c}, 1) \operatorname{Ro}_{2 \ell}^{(4)}\left(\mathrm{c}, \xi_{1}\right)-(1+\zeta) \operatorname{Ro}_{2 \ell}^{(4)^{\prime}}(\mathrm{c}, 1) \operatorname{Ro}_{2 \ell}^{(1)}\left(\mathrm{c}, \xi_{1}\right)},
\end{aligned}
$$

with

$$
\zeta=Z_{1} / Z_{2}
$$

and the prime indicates the derivative with respect to $\xi$.
Of particular interest are the surface current densities $\underline{J}$ on the metallic boundaries of the structure of Fig. 1. With E-polarization, these currents are parallel to the z-axis and given by:

$$
\begin{aligned}
J_{z} & =-H_{1 \xi} \text { on } D E(v=0), \\
& =H_{2 \xi} \text { on } D C(v=2 \pi), \\
& =H_{1 \xi} \text { on } O A(v=\pi / 2), \\
& =-H_{2 \xi} \text { on } O B(v=3 \pi / 2), \\
& =-H_{2 v} \text { on } B C\left(\xi=\xi_{1}\right),
\end{aligned}
$$

where:

$$
\begin{aligned}
\left.H_{1 \xi}\right|_{v=0}= & \frac{j 8}{c Z_{1}} \sqrt{\frac{2 \pi}{\xi^{2}-1}} \sum_{\ell=0}^{\infty} \frac{(-1)^{\ell}}{N_{2 \ell}^{(o)}}\left[\operatorname{Ro}_{2 \ell}^{(1)}(\mathrm{c}, \xi)+\right. \\
& \left.a_{\ell} \operatorname{Ro}_{2 \ell}^{(4)}(\mathrm{c}, \xi)\right] \mathrm{So}_{2 \ell}\left(\mathrm{c}, \cos \varphi_{0}\right)
\end{aligned}
$$

$$
\begin{aligned}
& \left.H_{2 \xi}\right|_{v=2 \pi}=\frac{j 8}{c Z_{2}} \sqrt{\frac{2 \pi}{\xi^{2}-1}} \sum_{\ell=0}^{\infty} \frac{(-1)^{\ell}}{N_{2 \ell}^{(o)}} a_{\ell}\left[\frac{\operatorname{Ro}_{2 \ell}^{(4)}\left(\mathrm{c}, \xi_{1}\right)}{\operatorname{Ro}_{2 \ell}^{(1)}\left(\mathrm{c}, \xi_{1}\right)} \times\right. \\
& \left.\operatorname{Ro}_{2 \ell}^{(1)}(\mathrm{c}, \xi)-\mathrm{Ro}_{2 \ell}^{(4)}(\mathrm{c}, \xi)\right] \operatorname{So}_{2 \ell}\left(\mathrm{c}, \cos \varphi_{0}\right),
\end{aligned}
$$

$$
\begin{aligned}
\left.H_{1 \xi}\right|_{v=\frac{\pi}{2}}= & \frac{j 8 \sqrt{2 \pi}}{c Z_{1} \xi} \sum_{\ell=0}^{\infty} \frac{(-1)^{\ell}}{N_{2 \ell}^{(o)}}\left[\operatorname{Ro}_{2 \ell}^{(1)}(\mathrm{c}, \xi)+\mathrm{a}_{\ell} \mathrm{Ro}_{2 \ell}^{(4)}(\mathrm{c}, \xi)\right] \times \\
& \mathrm{So}_{2 \ell}\left(\mathrm{c}, \cos \varphi_{0}\right)\left[\frac{\partial}{\partial \mathrm{v}} \mathrm{So}_{2 \ell}(\mathrm{c}, \eta)\right]_{\mathrm{v}=\frac{\pi}{2}}
\end{aligned}
$$

$$
\begin{aligned}
& \left.H_{2 \xi}\right|_{v=3 \pi / 2}=\frac{j 8 \sqrt{2 \pi}}{c Z_{2} \xi} \sum_{\ell=0}^{\infty} \frac{(-1)^{\ell}}{N_{2 \ell}^{(o)}} a_{\ell}\left[\frac{\mathrm{Ro}_{2 \ell}^{(4)}\left(\mathrm{c}, \xi_{1}\right)}{\operatorname{Ro}_{2 \ell}^{(1)}\left(\mathrm{c}, \xi_{1}\right)} \mathrm{Ro}_{2 \ell}^{(1)}(\mathrm{c}, \xi)\right. \\
& \left.-\mathrm{Ro}_{2 \ell}^{(4)}(\mathrm{c}, \xi)\right] \mathrm{So}_{2 \ell}\left(\mathrm{c}, \cos \varphi_{0}\right)\left[\frac{\partial}{\partial \mathrm{v}} \mathrm{So}_{2 \ell}(\mathrm{c}, \eta)\right]_{\mathrm{v}=3 \pi / 2},
\end{aligned}
$$

$$
\begin{aligned}
\left.H_{2 v}\right|_{\xi=\xi_{1}}= & \frac{8}{c Z_{2}} \sqrt{\frac{2 \pi}{\xi_{1}^{2}-\eta^{2}}} \sum_{\ell=0}^{\infty} \frac{(-1)^{\ell}}{N_{2 \ell}^{(o)}} \frac{a_{\ell}}{\operatorname{Ro}_{2 \ell}^{(1)}\left(\mathrm{c}, \xi_{1}\right)} \times \\
& \mathrm{So}_{2 \ell}(\mathrm{c}, \eta) \mathrm{So}_{2 \ell}\left(\mathrm{c}, \cos \varphi_{0}\right) .
\end{aligned}
$$

Within the quadrant $(x>0, y>0)$, the bistatic radar cross section (RCS) $\sigma_{E}(\varphi)$ of the partially covered trench is:

$$
\begin{aligned}
\frac{\sigma_{E}(\varphi)}{\lambda}= & 128 \pi\left|\sum_{\ell=0}^{\infty} \frac{a_{\ell}}{N_{2 \ell}^{(o)}} \operatorname{So}_{2 \ell}(\mathrm{c}, \cos \varphi) \operatorname{So}_{2 \ell}\left(\mathrm{c}, \cos \varphi_{0}\right)\right|^{2} \\
& 0<\varphi, \varphi_{0}<\pi / 2 .
\end{aligned}
$$

In the limit when the trench recedes to infinity $\left(\xi_{1} \rightarrow \infty\right.$ with $\operatorname{Im} \mathrm{c}<0$ ), the slit $\xi=1$ connects two isorefractive quarter spaces. In this case,

$$
\left.a_{\ell}\right|_{\xi_{1} \rightarrow \infty, \operatorname{Im} \mathrm{c}<0}=\frac{-1}{1+\zeta} \frac{\operatorname{Ro}_{2 \ell}^{(1)^{\prime}}(\mathrm{c}, 1)}{\operatorname{Ro}_{2 \ell}^{(4)^{\prime}}(\mathrm{c}, 1)},
$$


and the diffracted field in the quarter space $(x \geq 0, y \geq 0)$ becomes:

$$
\begin{aligned}
&\left.E_{1 z}^{d}\right|_{\xi_{1} \rightarrow \infty, \operatorname{Imc} c 0}= \frac{-8 \sqrt{2 \pi}}{1+\zeta} \sum_{\ell=0}^{\infty} \frac{(-1)^{\ell}}{N_{2 \ell}^{(o)}} \frac{\operatorname{Ro}_{2 \ell}^{(1)^{\prime}}(\mathrm{c}, 1)}{\operatorname{Ro}_{2 \ell}^{(4)^{\prime}}(\mathrm{c}, 1)} \times \\
& \operatorname{Ro}_{2 \ell}^{(4)}(\mathrm{c}, \xi) \operatorname{So}_{2 \ell}(\mathrm{c}, \eta) \operatorname{So}_{2 \ell}\left(\mathrm{c}, \cos \varphi_{0}\right)
\end{aligned}
$$

and is valid for $0 \leq v \leq \pi / 2$. The diffracted field $\left.E_{2 z}^{d}\right|_{\xi_{1} \rightarrow \infty, \operatorname{Imc}<0}$ in the quarter space $(x \geq 0, y \leq 0)$ is given by (28) multiplied by minus one, and is valid for $3 \pi / 2 \leq v \leq 2 \pi$.

\section{B. H-polarization}

For an incident plane wave with the magnetic field parallel to the z-axis,

$$
\underline{H}^{i}=\hat{z} H_{z}^{i}=\hat{z} e^{j k\left(x \cos \varphi_{0}+y \sin \varphi_{0}\right)},\left(0<\varphi_{0}<\pi / 2\right),
$$

the derivations are similar to those for E-polarization, hence only the results are given.

The total magnetic field $H_{1 z}$ in the quarter space $(x \geq$ $0, y \geq 0)$ is:

$$
H_{1 z}=H_{1 z}^{g o}+H_{1 z}^{d},
$$

where the geometric-optics field $H_{1 z}^{g o}$ (i.e., the field in the absence of the trench) is the sum of four plane waves:

$$
H_{1 z}^{g o}=8 \sqrt{2 \pi} \sum_{\ell=0}^{\infty} \frac{(-1)^{\ell}}{N_{2 \ell}^{(e)}} \operatorname{Re}_{2 \ell}^{(1)}(\mathrm{c}, \xi) \operatorname{Se}_{2 \ell}(\mathrm{c}, \eta) \operatorname{Se}_{2 \ell}\left(\mathrm{c}, \cos \varphi_{0}\right) ;
$$

only the even Mathieu functions of even order appear, because of the boundary conditions on the two metallic walls. The diffracted field due to the slotted trench is:

$H_{1 z}^{d}=8 \sqrt{2 \pi} \sum_{\ell=0}^{\infty} \frac{(-1)^{\ell}}{N_{2 \ell}^{(e)}} b_{\ell} \operatorname{Re}_{2 \ell}^{(4)}(\mathrm{c}, \xi) \operatorname{Se}_{2 \ell}(\mathrm{c}, \eta) \operatorname{Se}_{2 \ell}\left(\mathrm{c}, \cos \varphi_{0}\right)$.

The field inside the trench is

$$
\begin{array}{r}
H_{2 z}=8 \sqrt{2 \pi} \zeta \sum_{\ell=0}^{\infty} \frac{(-1)^{\ell}}{N_{2 \ell}^{(e)}} b_{\ell}\left[\frac{\operatorname{Re}_{2 \ell}^{(4)^{\prime}}\left(\mathrm{c}, \xi_{1}\right)}{\operatorname{Re}_{2 \ell}^{(1)^{\prime}}\left(\mathrm{c}, \xi_{1}\right)} \operatorname{Re}_{2 \ell}^{(1)}(\mathrm{c}, \xi)-\right. \\
\left.\operatorname{Re}_{2 \ell}^{(4)}(\mathrm{c}, \xi)\right] \operatorname{Se}_{2 \ell}(\mathrm{c}, \eta) \operatorname{Se}_{2 \ell}\left(\mathrm{c}, \cos \varphi_{0}\right),
\end{array}
$$

where $\zeta$ is given by (19), the prime means the derivative with respect to $\xi$, and

$$
\begin{aligned}
& b_{\ell}= \\
& \frac{\operatorname{Re}_{2 \ell}^{(1)}(\mathrm{c}, 1) \operatorname{Re}_{2 \ell}^{(1)^{\prime}}\left(\mathrm{c}, \xi_{1}\right)}{\zeta \operatorname{Re}_{2 \ell}^{(1)}(\mathrm{c}, 1) \operatorname{Re}_{2 \ell}^{(4)^{\prime}}\left(\mathrm{c}, \xi_{1}\right)-(1+\zeta) \operatorname{Re}_{2 \ell}^{(4)}(\mathrm{c}, 1) \operatorname{Re}_{2 \ell}^{(1)^{\prime}}\left(\mathrm{c}, \xi_{1}\right)} .
\end{aligned}
$$

The surface current density $\underline{J}$ on the metallic boundaries of Fig. 1 is given by:

$$
\begin{aligned}
\underline{J} & =\hat{\xi} H_{1 z} \text { on } D E(v=0), \\
& =-\hat{\xi} H_{2 z} \text { on } D C(v=2 \pi), \\
& =-\hat{\xi} H_{1 z} \text { on } O A(v=\pi / 2), \\
& =\hat{\xi} H_{2 z} \text { on } O B(v=3 \pi / 2), \\
& =\hat{v} H_{2 z} \text { on } B C\left(\xi=\xi_{1}\right),
\end{aligned}
$$

where:

$$
\begin{aligned}
\left.H_{1 z}\right|_{v=0}= & 8 \sqrt{2 \pi} \sum_{\ell=0}^{\infty} \frac{(-1)^{\ell}}{N_{2 \ell}^{(e)}}\left[\operatorname{Re}_{2 \ell}^{(1)}(\mathrm{c}, \xi)+\right. \\
& \left.b_{\ell} \operatorname{Re}_{2 \ell}^{(4)}(\mathrm{c}, \xi)\right] \operatorname{Se}_{2 \ell}\left(\mathrm{c}, \cos \varphi_{0}\right),
\end{aligned}
$$

$$
\begin{gathered}
\left.H_{2 z}\right|_{v=2 \pi}=8 \sqrt{2 \pi} \zeta \sum_{\ell=0}^{\infty} \frac{(-1)^{\ell}}{N_{2 \ell}^{(e)}} b_{\ell}\left[\frac{\operatorname{Re}_{2 \ell}^{(4)^{\prime}}\left(\mathrm{c}, \xi_{1}\right)}{\operatorname{Re}_{2 \ell}^{(1)^{\prime}}\left(\mathrm{c}, \xi_{1}\right)} \operatorname{Re}_{2 \ell}^{(1)}(\mathrm{c}, \xi)-\right. \\
\left.\operatorname{Re}_{2 \ell}^{(4)}(\mathrm{c}, \xi)\right] \operatorname{Se}_{2 \ell}\left(\mathrm{c}, \cos \varphi_{0}\right),
\end{gathered}
$$

$$
\left.H_{1 z}\right|_{v=\pi / 2}=8 \sqrt{2 \pi} \sum_{\ell=0}^{\infty} \frac{(-1)^{\ell}}{N_{2 \ell}^{(e)}}\left[\operatorname{Re}_{2 \ell}^{(1)}(\mathrm{c}, \xi)+\mathrm{b}_{\ell} \operatorname{Re}_{2 \ell}^{(4)}(\mathrm{c}, \xi)\right] \times
$$

$$
\mathrm{Se}_{2 \ell}\left(\mathrm{c}, \cos \varphi_{0}\right)\left[\mathrm{Se}_{2 \ell}(\mathrm{c}, \eta)\right]_{\mathrm{v}=\pi / 2} \text {, }
$$

$$
\begin{gathered}
\left.H_{2 z}\right|_{v=3 \pi / 2}=8 \sqrt{2 \pi} \zeta \sum_{\ell=0}^{\infty} \frac{(-1)^{\ell}}{N_{2 \ell}^{(e)}} b_{\ell}\left[\frac{\operatorname{Re}_{2 \ell}^{(4)^{\prime}}\left(\mathrm{c}, \xi_{1}\right)}{\operatorname{Re}_{2 \ell}^{(1)^{\prime}}\left(\mathrm{c}, \xi_{1}\right)} \operatorname{Re}_{2 \ell}^{(1)}(\mathrm{c}, \xi)-\right. \\
\left.\operatorname{Re}_{2 \ell}^{(4)}(\mathrm{c}, \xi)\right] \operatorname{Se}_{2 \ell}\left(\mathrm{c}, \cos \varphi_{0}\right)\left[\operatorname{Se}_{2 \ell}(\mathrm{c}, \eta)\right]_{\mathrm{v}=3 \pi / 2}
\end{gathered}
$$

$$
\left.H_{2 z}\right|_{\xi=\xi_{1}}=-j 8 \zeta \sqrt{\frac{2 \pi}{\xi_{1}^{2}-1}} \sum_{\ell=0}^{\infty} \frac{(-1)^{\ell}}{N_{2 \ell}^{(e)}} \frac{b_{\ell}}{\operatorname{Re}_{2 \ell}^{(1)^{\prime}}\left(\mathrm{c}, \xi_{1}\right)} \times
$$$$
\mathrm{Se}_{2 \ell}\left(\mathrm{c}, \cos \varphi_{0}\right) \mathrm{Se}_{2 \ell}(\mathrm{c}, \eta) \text {; }
$$

note that in (38) and (39), $\mathrm{Se}_{2 \ell}(\mathrm{c}, \eta)$ has the same value at $v=\pi / 2$ and $v=3 \pi / 2$.

The bistatic radar cross section $\sigma_{H}(\varphi)$ of the partially covered trench is:

$$
\begin{gathered}
\frac{\sigma_{H}(\varphi)}{\lambda}=128 \pi\left|\sum_{\ell=0}^{\infty} \frac{b_{\ell}}{N_{2 \ell}^{(e)}} \operatorname{Se}_{2 \ell}(\mathrm{c}, \cos \varphi) \operatorname{Se}_{2 \ell}\left(\mathrm{c}, \cos \varphi_{0}\right)\right|^{2} \\
0<\varphi, \varphi_{0}<\pi / 2 .
\end{gathered}
$$

In the limit when the trench recedes to infinity $\left(\xi_{1} \rightarrow \infty\right.$ with $\operatorname{Im} c<0$ ):

$$
\left.b_{\ell}\right|_{\xi_{1} \rightarrow \infty, \operatorname{Imc}<0}=\frac{-1}{1+\zeta} \frac{\operatorname{Re}_{2 \ell}^{(1)}(\mathrm{c}, 1)}{\operatorname{Re}_{2 \ell}^{(4)}(\mathrm{c}, 1)}
$$


and the diffracted field in the quarter space $(x \geq 0, y \geq 0)$ becomes:

$$
\begin{aligned}
&\left.H_{1 z}^{d}\right|_{\xi_{1} \rightarrow \infty, \operatorname{Imc}<0}=-\frac{8 \sqrt{2 \pi}}{1+\zeta} \sum_{\ell=0}^{\infty} \frac{(-1)^{\ell}}{N_{2 \ell}^{(e)}} \frac{\operatorname{Re}_{2 \ell}^{(1)}(\mathrm{c}, 1)}{\operatorname{Re}_{2 \ell}^{(4)}(\mathrm{c}, 1)} \times \\
& \operatorname{Re}_{2 \ell}^{(4)}(\mathrm{c}, \xi) \operatorname{Se}_{2 \ell}\left(\mathrm{c}, \cos \varphi_{0}\right) \operatorname{Se}_{2 \ell}(\mathrm{c}, \eta)
\end{aligned}
$$

and is valid for $0 \leq v \leq \pi / 2$. The diffracted field $\left.H_{2 z}\right|_{\xi_{1} \rightarrow \infty, \operatorname{Imc}<0}$ in the quarter space $(x \geq 0, y \leq 0)$ is given by (43) multiplied by $-\zeta$, and is valid in the range $3 \pi / 2 \leq v \leq 2 \pi$.

\section{LINE SOURCE INCIDENCE}

\section{A. E-polarization}

Consider an electric line source parallel to the z-axis and located outside the trench at $\left(x_{0}, y_{0}\right) \equiv\left(u_{0}, v_{0}\right)$, where $x_{0}>0, y_{0}>0$, whose primary electric field is

$$
\underline{E}^{i}=\hat{z} E_{z}^{i}=\hat{z} H_{0}^{(2)}(k R)
$$

where

$$
R=\sqrt{\left(x-x_{0}\right)^{2}+\left(y-y_{0}\right)^{2}}
$$

is the distance between the line source and the observation point $(x, y) \equiv(u, v)$. The incident field may be expanded in a series of elliptic-cylinder functions [2]:

$$
\begin{aligned}
& E_{1 z}^{i}=H_{0}^{(2)}(k R)= \\
& 4 \sum_{m=0}^{\infty}\left[\frac{1}{N_{m}^{(e)}} \operatorname{Re}_{\mathrm{m}}^{(1)}\left(\mathrm{c}, \xi_{<}\right) \operatorname{Re}_{\mathrm{m}}^{(4)}\left(\mathrm{c}, \xi_{>}\right) \operatorname{Se}_{\mathrm{m}}\left(\mathrm{c}, \eta_{0}\right) \operatorname{Se}_{\mathrm{m}}(\mathrm{c}, \eta)+\right. \\
& \left.\frac{1}{N_{m}^{(o)}} \operatorname{Ro}_{\mathrm{m}}^{(1)}\left(\mathrm{c}, \xi_{<}\right) \operatorname{Ro}_{\mathrm{m}}^{(4)}\left(\mathrm{c}, \xi_{>}\right) \operatorname{So}_{\mathrm{m}}\left(\mathrm{c}, \eta_{0}\right) \operatorname{So}_{\mathrm{m}}(\mathrm{c}, \eta)\right]
\end{aligned}
$$

where $\xi_{<}\left(\xi_{>}\right)$is the smaller (larger) between $\xi$ and $\xi_{0}$.

In the quarter-space $(x \geq 0, y \geq 0)$ outside the trench, the total electric field $E_{1 z}$ may be written as the sum of two terms, as in (13). The geometrical-optics field $E_{1 z}^{g o}$ is the total field that would be present in the absence of the trench, and is the sum of the fields due to four line sources, i.e. the primary line $S$ and its three images $S_{1}, S_{2}$ and $S_{3}$ (see Fig. 3):

$$
E_{1 z}^{g o}=H_{0}^{(2)}(k R)-H_{0}^{(2)}\left(k R_{1}\right)-H_{0}^{(2)}\left(k R_{2}\right)+H_{0}^{(2)}\left(k R_{3}\right),
$$

where:

$$
\begin{aligned}
& R_{1}=\sqrt{\left(x-x_{0}\right)^{2}+\left(y+y_{0}\right)^{2}}, \\
& R_{2}=\sqrt{\left(x+x_{0}\right)^{2}+\left(y-y_{0}\right)^{2}}, \\
& R_{3}=\sqrt{\left(x+x_{0}\right)^{2}+\left(y+y_{0}\right)^{2}} .
\end{aligned}
$$

The image line $S_{1}$ is located at $\left(x_{0},-y_{0}\right) \equiv\left(u_{0}, 2 \pi-v_{0}\right)$, the image line $S_{2}$ at $\left(-x_{0}, y_{0}\right) \equiv\left(u_{0}, \pi-v o\right)$, and the image line $S_{3}$ at $\left(-x_{0},-y_{0}\right) \equiv\left(u_{0}, \pi+v_{0}\right)$. By expanding the Hankel functions in (47) into series of the type (46) and

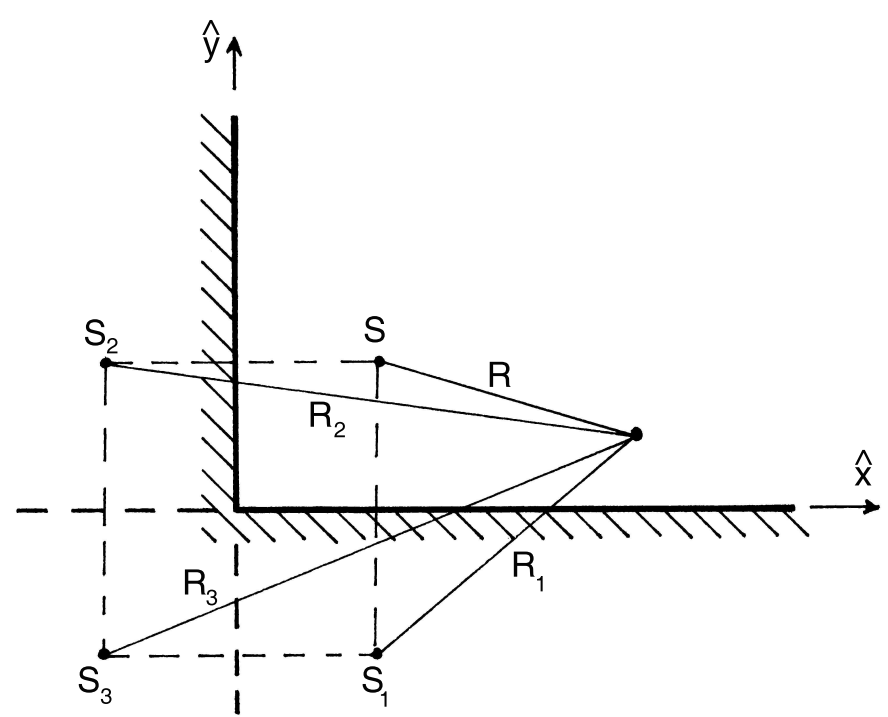

Fig. 3. Geometric-optics contribution for line-source excitation

utilizing properties of the angular Mathieu functions, it is found that

$$
\begin{aligned}
& E_{1 z}^{g o}=16 \times \\
& \sum_{l=0}^{\infty} \frac{1}{N_{2 \ell}^{(o)}} \mathrm{Ro}_{2 \ell}^{(1)}\left(\mathrm{c}, \xi_{<}\right) \mathrm{Ro}_{2 \ell}^{(4)}\left(\mathrm{c}, \xi_{>}\right) \operatorname{So}_{2 \ell}\left(\mathrm{c}, \eta_{0}\right) \operatorname{So}_{2 \ell}(\mathrm{c}, \eta),
\end{aligned}
$$

which involves only odd Mathieu functions of even order. The diffracted portion $E_{1 z}^{d}$ of the field in the quadrant $(x \geq 0, y \geq 0)$, due to the presence of the trench, and the total field $E_{2 z}$ inside the trench, are similarly given by infinite series of odd Mathieu functions of even order, whose modal coefficients are determined by imposing the boundary conditions. It is found that

$$
\begin{aligned}
& E_{1 z}^{d}=16 \times \\
& \sum_{l=0}^{\infty} \frac{a_{\ell}}{N_{2 \ell}^{(o)}} \mathrm{Ro}_{2 \ell}^{(4)}\left(\mathrm{c}, \xi_{0}\right) \mathrm{Ro}_{2 \ell}^{(4)}(\mathrm{c}, \xi) \operatorname{So}_{2 \ell}\left(\mathrm{c}, \eta_{0}\right) \mathrm{So}_{2 \ell}(\mathrm{c}, \eta),
\end{aligned}
$$

$$
\begin{gathered}
E_{2 z}=16 \sum_{l=0}^{\infty} \frac{a_{l}}{N_{2 \ell}^{(o)}} \operatorname{Ro}_{2 \ell}^{(4)}\left(\mathrm{c}, \xi_{0}\right)\left[\frac{\mathrm{Ro}_{2 \ell}^{(4)}\left(\mathrm{c}, \xi_{1}\right)}{\operatorname{Ro}_{2 \ell}^{(1)}\left(\mathrm{c}, \xi_{1}\right)} \mathrm{Ro}_{2 \ell}^{(1)}(\mathrm{c}, \xi)-\right. \\
\left.\operatorname{Ro}_{2 \ell}^{(4)}(\mathrm{c}, \xi)\right] \operatorname{So}_{2 \ell}\left(\mathrm{c}, \eta_{0}\right) \operatorname{So}_{2 \ell}(\mathrm{c}, \eta)
\end{gathered}
$$

where $a_{\ell}$ is given by (18).

The surface current densities on the metal boundaries are still given by (20) with:

$$
\begin{array}{r}
\left.H_{1 \xi}\right|_{v=0}=\frac{16 j}{c Z_{1} \sqrt{\xi^{2}-1}} \sum_{l=0}^{\infty} \frac{1}{N_{2 \ell}^{(o)}}\left[\operatorname{Ro}_{2 \ell}^{(1)}\left(\mathrm{c}, \xi_{<}\right)+\right. \\
\left.a_{\ell} \operatorname{Ro}_{2 \ell}^{(4)}\left(\mathrm{c}, \xi_{<}\right)\right] \operatorname{Ro}_{2 \ell}^{(4)}\left(\mathrm{c}, \xi_{>}\right) \operatorname{So}_{2 \ell}\left(\mathrm{c}, \eta_{0}\right),
\end{array}
$$




$$
\begin{gathered}
\left.H_{2 \xi}\right|_{v=2 \pi}=\frac{16 j}{c Z_{2} \sqrt{\xi^{2}-1}} \sum_{l=0}^{\infty} \frac{a_{\ell}}{N_{2 \ell}^{(o)}} \operatorname{Ro}_{2 \ell}^{(4)}\left(\mathrm{c}, \xi_{0}\right) \times \\
{\left[\frac{\mathrm{Ro}_{2 \ell}^{(4)}\left(\mathrm{c}, \xi_{1}\right)}{\operatorname{Ro}_{2 \ell}^{(1)}\left(\mathrm{c}, \xi_{1}\right)} \mathrm{Ro}_{2 \ell}^{(1)}(\mathrm{c}, \xi)-\mathrm{Ro}_{2 \ell}^{(4)}(\mathrm{c}, \xi)\right] \operatorname{So}_{2 \ell}\left(\mathrm{c}, \eta_{0}\right), \quad(55)} \\
\left.H_{1 \xi}\right|_{v=\pi / 2}=\frac{16 j}{c Z_{1} \xi} \sum_{l=0}^{\infty} \frac{1}{N_{2 \ell}^{(o)}}\left[\operatorname{Ro}_{2 \ell}^{(1)}\left(\mathrm{c}, \xi_{<}\right)+\mathrm{a}_{\ell} \operatorname{Ro}_{2 \ell}^{(4)}\left(\mathrm{c}, \xi_{<}\right)\right] \times \\
\operatorname{Ro}_{2 \ell}^{(4)}\left(\mathrm{c}, \xi_{>}\right) \operatorname{So}_{2 \ell}\left(\mathrm{c}, \eta_{0}\right)\left[\frac{\partial}{\partial \mathrm{v}} \operatorname{So}_{2 \ell}(\mathrm{c}, \eta)\right]_{\mathrm{v}=\pi / 2}, \\
\left.H_{2 \xi}\right|_{v=3 \pi / 2}=\frac{16 j}{c Z_{2} \xi} \sum_{l=0}^{\infty} \frac{a_{\ell}}{N_{2 \ell}^{(o)}} \operatorname{Ro}_{2 \ell}^{(4)}\left(\mathrm{c}, \xi_{0}\right)\left[\frac{\mathrm{Ro}_{2 \ell}^{(4)}\left(\mathrm{c}, \xi_{1}\right)}{\operatorname{Ro}_{2 \ell}^{(1)}\left(\mathrm{c}, \xi_{1}\right)} \times\right. \\
\left.\operatorname{Ro}_{2 \ell}^{(1)}(\mathrm{c}, \xi)-\mathrm{Ro}_{2 \ell}^{(4)}(\mathrm{c}, \xi)\right] \operatorname{So}_{2 \ell}\left(\mathrm{c}, \eta_{0}\right)\left[\frac{\partial}{\partial \mathrm{v}} \operatorname{So}_{2 \ell}(\mathrm{c}, \eta)\right]_{\mathrm{v}=3 \pi / 2}
\end{gathered}
$$

$$
\begin{array}{r}
\left.H_{2 v}\right|_{\xi=\xi_{1}}=\frac{16}{c Z_{2} \sqrt{\xi_{1}^{2}-\eta^{2}}} \sum_{l=0}^{\infty} \frac{a_{\ell}}{N_{2 \ell}^{(o)}} \frac{\operatorname{Ro}_{2 \ell}^{(4)}\left(\mathrm{c}, \xi_{0}\right)}{\operatorname{Ro}_{2 \ell}^{(1)}\left(\mathrm{c}, \xi_{1}\right)} \times \\
\operatorname{So}_{2 \ell}\left(\mathrm{c}, \eta_{0}\right) \operatorname{So}_{2 \ell}(\mathrm{c}, \eta) .
\end{array}
$$

If the trench recedes to infinity $\left(\xi_{1} \rightarrow \infty\right.$ with Im $\mathrm{c}<$ $0)$, then the slit $\xi=1$ connects two isorefractive quarter spaces. Use of (27) in (52) and (53) yields:

$$
\begin{gathered}
\left.E_{1 z}^{d}\right|_{\xi_{1} \rightarrow \infty, \mathrm{Im} \mathrm{c}<0}=\frac{-16}{1+\zeta} \sum_{l=0}^{\infty} \frac{1}{N_{2 \ell}^{(o)}} \frac{\mathrm{Ro}_{2 \ell}^{(1)^{\prime}}(\mathrm{c}, 1)}{\operatorname{Ro}_{2 \ell}^{(4)^{\prime}}(\mathrm{c}, 1)} \times \\
\operatorname{Ro}_{2 \ell}^{(4)}\left(\mathrm{c}, \xi_{0}\right) \operatorname{Ro}_{2 \ell}^{(4)}(\mathrm{c}, \xi) \operatorname{So}_{2 \ell}\left(\mathrm{c}, \eta_{0}\right) \operatorname{So}_{2 \ell}(\mathrm{c}, \eta),
\end{gathered}
$$

valid for $0 \leq v \leq \pi / 2$. The field $\left.E_{2 z}\right|_{\xi_{1} \rightarrow \infty, \operatorname{Im} \mathrm{c}<0}$ is given by (59) multiplied by minus one, and is valid for $3 \pi / 2 \leq$ $v \leq 2 \pi$.

Let us now consider the case when the electric line source (44) is located inside the trench, i.e. $1 \leq \xi_{0}<\xi_{1}$ and $3 \pi / 2<v_{0}<2 \pi$. The total electric field inside the trench is:

$$
E_{2 z}=E_{2 z}^{g o}+E_{2 z}^{d}
$$

where $E_{2 z}^{g o}$ is the geometric-optics field due to the line source in the presence of a $90^{\circ}$ metallic corner reflector, and is still given by the right-hand side of (51), whereas the diffracted field component $E_{2 z}^{d}$ accounts for the slot at $\xi=1$ as well as the elliptic metal wall at $\xi=\xi_{1}$, and may be written as:

$$
\begin{array}{r}
E_{2 z}^{d}=16 \sum_{l=0}^{\infty} \frac{1}{N_{2 \ell}^{(o)}}\left[\tilde{a}_{\ell} \operatorname{Ro}_{2 \ell}^{(1)}(\mathrm{c}, \xi)+\tilde{\mathrm{c}}_{\ell} \mathrm{Ro}_{2 \ell}^{(4)}(\mathrm{c}, \xi)\right] \times \\
\mathrm{So}_{2 \ell}\left(\mathrm{c}, \eta_{0}\right) \mathrm{So}_{2 \ell}(\mathrm{c}, \eta) .
\end{array}
$$

The total field $E_{1 z}$ in the quadrant $(x \geq 0, y \geq 0)$ is:

$$
E_{1 z}=-16 \sum_{l=0}^{\infty} \frac{\tilde{c}_{\ell}}{N_{2 \ell}^{(o)}} \operatorname{Ro}_{2 \ell}^{(4)}(\mathrm{c}, \xi) \operatorname{So}_{2 \ell}\left(\mathrm{c}, \eta_{0}\right) \operatorname{So}_{2 \ell}(\mathrm{c}, \eta)
$$

and the modal coefficients $\tilde{a}_{\ell}$ and $\tilde{c}_{\ell}$ are explicitly determined by imposing the boundary conditions:

$$
\begin{array}{r}
\tilde{a_{\ell}}=-\frac{\operatorname{Ro}_{2 \ell}^{(4)}\left(\mathrm{c}, \xi_{1}\right)}{\Delta_{\ell}^{(o)}}\left[\operatorname{Ro}_{2 \ell}^{(1)^{\prime}}(\mathrm{c}, 1) \operatorname{Ro}_{2 \ell}^{(4)}\left(\mathrm{c}, \xi_{0}\right)-\right. \\
\left.\left(1+\zeta^{-1}\right) \operatorname{Ro}_{2 \ell}^{(4)^{\prime}}(\mathrm{c}, 1) \operatorname{Ro}_{2 \ell}^{(1)}\left(\mathrm{c}, \xi_{0}\right)\right], \\
\tilde{c_{\ell}}=-\frac{\operatorname{Ro}_{2 \ell}^{(1)^{\prime}}(\mathrm{c}, 1)}{\Delta_{\ell}^{(o)}}\left[\operatorname{Ro}_{2 \ell}^{(1)}\left(\mathrm{c}, \xi_{0}\right) \operatorname{Ro}_{2 \ell}^{(4)}\left(\mathrm{c}, \xi_{1}\right)-\right. \\
\left.\operatorname{Ro}_{2 \ell}^{(1)}\left(\mathrm{c}, \xi_{1}\right) \operatorname{Ro}_{2 \ell}^{(4)}\left(\mathrm{c}, \xi_{0}\right)\right],
\end{array}
$$

where

$$
\begin{aligned}
\Delta_{\ell}^{(o)}= & \operatorname{Ro}_{2 \ell}^{(1)^{\prime}}(\mathrm{c}, 1) \operatorname{Ro}_{2 \ell}^{(4)}\left(\mathrm{c}, \xi_{1}\right)- \\
& \left(1+\zeta^{-1}\right) \operatorname{Ro}_{2 \ell}^{(4)^{\prime}}(\mathrm{c}, 1) \operatorname{Ro}_{2 \ell}^{(1)}\left(\mathrm{c}, \xi_{1}\right) .
\end{aligned}
$$

The surface current densities on the metal boundaries are given by (20) with:

$$
\left.H_{1 \xi}\right|_{v=0}=\frac{-16 j}{c Z_{1} \sqrt{\xi^{2}-1}} \sum_{l=0}^{\infty} \frac{\tilde{c}_{\ell}}{N_{2 \ell}^{(o)}} \operatorname{Ro}_{2 \ell}^{(4)}(\mathrm{c}, \xi) \operatorname{So}_{2 \ell}\left(\mathrm{c}, \eta_{0}\right),
$$

$$
\begin{gathered}
\left.H_{2 \xi}\right|_{v=2 \pi}=\frac{-16 j}{c Z_{2} \sqrt{\xi^{2}-1}} \sum_{l=0}^{\infty} \frac{1}{N_{2 \ell}^{(o)}}\left[\operatorname{Ro}_{2 \ell}^{(1)}\left(\mathrm{c}, \xi_{<}\right) \operatorname{Ro}_{2 \ell}^{(4)}\left(\mathrm{c}, \xi_{>}\right)\right. \\
\left.+\tilde{a}_{\ell} \operatorname{Ro}_{2 \ell}^{(1)}(\mathrm{c}, \xi)+\tilde{\mathrm{c}}_{\ell} \operatorname{Ro}_{2 \ell}^{(4)}(\mathrm{c}, \xi)\right] \operatorname{So}_{2 \ell}\left(\mathrm{c}, \eta_{0}\right), \\
\left.H_{1 \xi}\right|_{v=\pi / 2}=\frac{16 j}{c Z_{1} \xi} \sum_{l=0}^{\infty} \frac{\tilde{c}_{\ell}}{N_{2 \ell}^{(o)}} \operatorname{Ro}_{2 \ell}^{(4)}(\mathrm{c}, \xi) \operatorname{So}_{2 \ell}\left(\mathrm{c}, \eta_{0}\right) \times \\
{\left[\frac{\partial}{\partial v} \operatorname{So}_{2 \ell}(\mathrm{c}, \eta)\right]_{v=\pi / 2},}
\end{gathered}
$$

$$
\left.H_{2 \xi}\right|_{v=3 \pi / 2}=\frac{16 j}{c Z_{2} \xi} \sum_{l=0}^{\infty} \frac{1}{N_{2 \ell}^{(o)}}\left[\operatorname{Ro}_{2 \ell}^{(1)}\left(\mathrm{c}, \xi_{<}\right) \operatorname{Ro}_{2 \ell}^{(4)}\left(\mathrm{c}, \xi_{>}\right)+\right.
$$$$
\left.\tilde{a}_{\ell} \operatorname{Ro}_{2 \ell}^{(1)}(\mathrm{c}, \xi)+\tilde{\mathrm{c}}_{\ell} \mathrm{Ro}_{2 \ell}^{(4)}(\mathrm{c}, \xi)\right] \mathrm{So}_{2 \ell}\left(\mathrm{c}, \eta_{0}\right)\left[\frac{\partial}{\partial \mathrm{v}} \mathrm{So}_{2 \ell}(\mathrm{c}, \eta)\right]_{\mathrm{v}=3 \pi / 2},
$$

$$
\begin{aligned}
& \left.H_{2 v}\right|_{\xi=\xi_{1}}=\frac{-16 j}{c Z_{2}} \sqrt{\frac{\xi_{1}^{2}-1}{\xi_{1}^{2}-\eta^{2}}} \sum_{l=0}^{\infty} \frac{1}{N_{2 \ell}^{(o)}}\left\{\tilde{a}_{\ell} \operatorname{Ro}_{2 \ell}^{(1)^{\prime}}\left(\mathrm{c}, \xi_{1}\right)+\right. \\
& \left.\left[\operatorname{Ro}_{2 \ell}^{(1)}\left(\mathrm{c}, \xi_{0}\right)+\tilde{c}_{\ell}\right] \operatorname{Ro}_{2 \ell}^{(4)^{\prime}}\left(\mathrm{c}, \xi_{1}\right)\right\} \operatorname{So}_{2 \ell}\left(\mathrm{c}, \eta_{0}\right) \operatorname{So}_{2 \ell}(\mathrm{c}, \eta) .
\end{aligned}
$$

\section{B. H-polarization}

The derivations are similar to those for E-polarization, hence only the results are given. For a magnetic line source 
parallel to the z-axis and located outside the trench at $(x \geq$ $0, y \geq o)$, whose primary magnetic field is

$$
\underline{H}^{i}=\hat{z} H_{z}^{i}=\hat{z} H_{0}^{(2)}(k R)
$$

with $R$ given by (45), the total magnetic field $H_{1 z}$ in the quarter-space $(x \geq 0, y \geq 0)$ is given by (30), where, with reference to Fig. 3:

$\left.\left.H_{1 z}^{g o}=H_{0}^{(2)}(k R)+H_{0}^{(2)}\left(k R_{1}\right)+H_{0}^{(2)}\left(k R_{2}\right)+H_{0}^{(2)}\right) k R_{3}\right)=$ $16 \sum_{l=0}^{\infty} \frac{1}{N_{2 \ell}^{(e)}} \operatorname{Re}_{2 \ell}^{(1)}\left(\mathrm{c}, \xi_{<}\right) \operatorname{Re}_{2 \ell}^{(4)}\left(\mathrm{c}, \xi_{>}\right) \operatorname{Se}_{2 \ell}\left(\mathrm{c}, \eta_{0}\right) \operatorname{Se}_{2 \ell}(\mathrm{c}, \eta)$.

The diffracted field $H_{1 z}^{d}$ in $(x \geq 0, y \geq 0)$ and the total field $H_{2 z}$ inside the trench are given by:

$$
\begin{gathered}
H_{1 z}^{d}=16 \sum_{l=0}^{\infty} \frac{b_{\ell}}{N_{2 \ell}^{(e)}} \operatorname{Re}_{2 \ell}^{(4)}\left(\mathrm{c}, \xi_{0}\right) \operatorname{Re}_{2 \ell}^{(4)}(\mathrm{c}, \xi) \operatorname{Se}_{2 \ell}\left(\mathrm{c}, \eta_{0}\right) \operatorname{Se}_{2 \ell}(\mathrm{c}, \eta), \\
H_{2 z}=16 \zeta \sum_{l=0}^{\infty} \frac{b_{\ell}}{N_{2 \ell}^{(e)}} \operatorname{Re}_{2 \ell}^{(4)}\left(\mathrm{c}, \xi_{0}\right)\left[\frac{\operatorname{Re}_{2 \ell}^{(4)^{\prime}}\left(\mathrm{c}, \xi_{1}\right)}{\operatorname{Re}_{2 \ell}^{(1)^{\prime}}\left(\mathrm{c}, \xi_{1}\right)} \operatorname{Re}_{2 \ell}^{(1)}(\mathrm{c}, \xi)\right. \\
\left.-\operatorname{Re}_{2 \ell}^{(4)}(\mathrm{c}, \xi)\right] \operatorname{Se}_{2 \ell}\left(\mathrm{c}, \eta_{0}\right) \operatorname{Se}_{2 \ell}(\mathrm{c}, \eta),
\end{gathered}
$$

where $b_{\ell}$ is given by (34). The surface current density on the metallic boundaries is given by (35) with

$$
\begin{gathered}
\left.H_{1 z}\right|_{v=0}=16 \sum_{l=0}^{\infty} \frac{1}{N_{2 \ell}^{(e)}}\left[\operatorname{Re}_{2 \ell}^{(1)}\left(\mathrm{c}, \xi_{<}\right)+\mathrm{b}_{\ell} \operatorname{Re}_{2 \ell}^{(4)}\left(\mathrm{c}, \xi_{<}\right)\right] \times \\
\operatorname{Re}_{2 \ell}^{(4)}\left(\mathrm{c}, \xi_{>}\right) \operatorname{Se}_{2 \ell}\left(\mathrm{c}, \eta_{0}\right), \\
\left.H_{1 z}\right|_{v=\pi / 2}=16 \sum_{l=0}^{\infty} \frac{1}{N_{2 \ell}^{(e)}}\left[\operatorname{Re}_{2 \ell}^{(1)}\left(\mathrm{c}, \xi_{<}\right)+\mathrm{b}_{\ell} \operatorname{Re}_{2 \ell}^{(4)}\left(\mathrm{c}, \xi_{<}\right)\right] \times \\
\operatorname{Re}_{2 \ell}^{(4)}\left(\mathrm{c}, \xi_{>}\right) \operatorname{Se}_{2 \ell}\left(\mathrm{c}, \eta_{0}\right)\left[\operatorname{Se}_{2 \ell}(\mathrm{c}, \eta)\right]_{\mathrm{v}=\pi / 2}, \\
\left.H_{2 z}\right|_{v=3 \pi / 2}=16 \zeta \sum_{l=0}^{\infty} \frac{b_{\ell}}{N_{2 \ell}^{(e)}} \operatorname{Re}_{2 \ell}^{(4)}\left(\mathrm{c}, \xi_{0}\right)\left[\frac{\operatorname{Re}_{2 \ell}^{(4)^{\prime}}\left(\mathrm{c}, \xi_{1}\right)}{\operatorname{Re}_{2 \ell}^{(1)^{\prime}}\left(\mathrm{c}, \xi_{1}\right)} \times\right. \\
\left.\operatorname{Re}_{2 \ell}^{(1)}(\mathrm{c}, \xi)-\operatorname{Re}_{2 \ell}^{(4)}(\mathrm{c}, \xi)\right] \operatorname{Se}_{2 \ell}\left(\mathrm{c}, \eta_{0}\right)\left[\operatorname{Se}_{2 \ell}(\mathrm{c}, \eta)\right]_{\mathrm{v}=3 \pi / 2},
\end{gathered}
$$

$$
\begin{aligned}
&\left.H_{2 z}\right|_{v=2 \pi}= 16 \zeta \sum_{l=0}^{\infty} \frac{b_{\ell}}{N_{2 \ell}^{(e)}} \operatorname{Re}_{2 \ell}^{(4)}\left(\mathrm{c}, \xi_{0}\right)\left[\frac{\operatorname{Re}_{2 \ell}^{(4)^{\prime}}\left(\mathrm{c}, \xi_{1}\right)}{\operatorname{Re}_{2 \ell}^{(1)^{\prime}}\left(\mathrm{c}, \xi_{1}\right)} \times\right. \\
&\left.\operatorname{Re}_{2 \ell}^{(1)}(\mathrm{c}, \xi)-\operatorname{Re}_{2 \ell}^{(4)}(\mathrm{c}, \xi)\right] \operatorname{Se}_{2 \ell}\left(\mathrm{c}, \eta_{0}\right)
\end{aligned}
$$

$$
\begin{aligned}
& \left.H_{2 z}\right|_{\xi=\xi_{1}}= \\
& \frac{-16 j \zeta}{\sqrt{\xi_{1}^{2}-1}} \sum_{l=0}^{\infty} \frac{b_{\ell}}{N_{2 \ell}^{(e)}} \frac{\operatorname{Re}_{2 \ell}^{(4)}\left(\mathrm{c}, \xi_{0}\right)}{\operatorname{Re}_{2 \ell}^{(1)^{\prime}}\left(\mathrm{c}, \xi_{1}\right)} \operatorname{Se}_{2 \ell}\left(\mathrm{c}, \eta_{0}\right) \operatorname{Se}_{2 \ell}(\mathrm{c}, \eta) .
\end{aligned}
$$

If the magnetic line source (71) is located inside the trench, then the total magnetic field inside the trench is

$$
H_{2 z}=H_{2 z}^{g o}+H_{2 z}^{d}
$$

and outside the trench (i.e., $x \geq 0, y \geq 0$ ) is $H_{1 z}$, where $H_{2 z}^{g o}$ is given by the right-hand side of (72) and

$$
\begin{aligned}
H_{2 z}^{d}=16 \sum_{l=0}^{\infty} \frac{1}{N_{2 \ell}^{(e)}}\left[\tilde{b}_{\ell} \operatorname{Re}_{2 \ell}^{(1)}(\mathrm{c}, \xi)+\tilde{\mathrm{d}}_{\ell} \operatorname{Re}_{2 \ell}^{(4)}(\mathrm{c}, \xi)\right] \times \\
\operatorname{Se}_{2 \ell}\left(\mathrm{c}, \eta_{0}\right) \operatorname{Se}_{2 \ell}(\mathrm{c}, \eta), \\
H_{1 z}=-16 \zeta^{-1} \sum_{l=0}^{\infty} \frac{\tilde{d}_{\ell}}{N_{2 \ell}^{(e)}} \operatorname{Re}_{2 \ell}^{(4)}(\mathrm{c}, \xi) \operatorname{Se}_{2 \ell}\left(\mathrm{c}, \eta_{0}\right) \operatorname{Se}_{2 \ell}(\mathrm{c}, \eta),
\end{aligned}
$$

where

$$
\begin{gathered}
\tilde{b}_{\ell}=-\frac{\operatorname{Re}_{2 \ell}^{(4)^{\prime}}\left(\mathrm{c}, \xi_{1}\right)}{\Delta_{\ell}^{(e)}}\left[\operatorname{Re}_{2 \ell}^{(1)}(\mathrm{c}, 1) \operatorname{Re}_{2 \ell}^{(4)}\left(\mathrm{c}, \xi_{0}\right)-\right. \\
\left.\left(1+\zeta^{-1}\right) \operatorname{Re}_{2 \ell}^{(4)}(\mathrm{c}, 1) \operatorname{Re}_{2 \ell}^{(1)}\left(\mathrm{c}, \xi_{0}\right)\right] \\
\tilde{d}_{\ell}=-\frac{\operatorname{Re}_{2 \ell}^{(1)}(\mathrm{c}, 1)}{\Delta_{\ell}^{(e)}}\left[\operatorname{Re}_{2 \ell}^{(1)}\left(\mathrm{c}, \xi_{0}\right) \operatorname{Re}_{2 \ell}^{(4)^{\prime}}\left(\mathrm{c}, \xi_{1}\right)-\right. \\
\left.\operatorname{Re}_{2 \ell}^{(1)^{\prime}}\left(\mathrm{c}, \xi_{1}\right) \operatorname{Re}_{2 \ell}^{(4)}\left(\mathrm{c}, \xi_{0}\right)\right]
\end{gathered}
$$

with

$$
\begin{aligned}
\Delta_{\ell}^{(e)}= & \operatorname{Re}_{2 \ell}^{(1)}(\mathrm{c}, 1) \operatorname{Re}_{2 \ell}^{(4)^{\prime}}\left(\mathrm{c}, \xi_{1}\right)- \\
& \left(1+\zeta^{-1}\right) \operatorname{Re}_{2 \ell}^{(4)}(\mathrm{c}, 1) \operatorname{Re}_{2 \ell}^{(1)^{\prime}}\left(\mathrm{c}, \xi_{1}\right) .
\end{aligned}
$$

The surface current density on the metal boundaries is given by (35) with

$$
\begin{gathered}
\left.H_{1 z}\right|_{v=0}=-16 \zeta^{-1} \sum_{l=0}^{\infty} \frac{\tilde{d}_{\ell}}{N_{2 \ell}^{(e)}} \operatorname{Re}_{2 \ell}^{(4)}(\mathrm{c}, \xi) \operatorname{Se}_{2 \ell}\left(\mathrm{c}, \eta_{0}\right), \\
\left.H_{1 z}\right|_{v=\pi / 2}=-16 \zeta^{-1} \sum_{l=0}^{\infty} \frac{\tilde{d}_{\ell}}{N_{2 \ell}^{(e)}} \operatorname{Re}_{2 \ell}^{(4)}(\mathrm{c}, \xi) \operatorname{Se}_{2 \ell}\left(\mathrm{c}, \eta_{0}\right) \times \\
{\left[\operatorname{Se}_{2 \ell}(\mathrm{c}, \eta)\right]_{v=\pi / 2},}
\end{gathered}
$$

$$
\begin{aligned}
& \left.H_{2 z}\right|_{v=3 \pi / 2}=16 \sum_{l=0}^{\infty} \frac{1}{N_{2 \ell}^{(e)}}\left[\operatorname{Re}_{2 \ell}^{(1)}\left(\mathrm{c}, \xi_{<}\right) \operatorname{Re}_{2 \ell}^{(4)}\left(\mathrm{c}, \xi_{>}\right)+\right. \\
& \left.\tilde{b}_{\ell} \operatorname{Re}_{2 \ell}^{(1)}(\mathrm{c}, \xi)+\tilde{\mathrm{d}}_{\ell} \operatorname{Re}_{2 \ell}^{(4)}(\mathrm{c}, \xi)\right] \operatorname{Se}_{2 \ell}\left(\mathrm{c}, \eta_{0}\right)\left[\operatorname{Se}_{2 \ell}(\mathrm{c}, \eta)\right]_{\mathrm{v}=3 \pi / 2}
\end{aligned}
$$

$$
\begin{array}{r}
\left.H_{2 z}\right|_{v=2 \pi}=16 \sum_{l=0}^{\infty} \frac{1}{N_{2 \ell}^{(e)}}\left[\operatorname{Re}_{2 \ell}^{(1)}\left(\mathrm{c}, \xi_{<}\right) \operatorname{Re}_{2 \ell}^{(4)}\left(\mathrm{c}, \xi_{>}\right)+\right. \\
\left.\tilde{b}_{\ell} \operatorname{Re}_{2 \ell}^{(1)}(\mathrm{c}, \xi)+\tilde{\mathrm{d}}_{\ell} \operatorname{Re}_{2 \ell}^{(4)}(\mathrm{c}, \xi)\right] \operatorname{Se}_{2 \ell}\left(\mathrm{c}, \eta_{0}\right)
\end{array}
$$




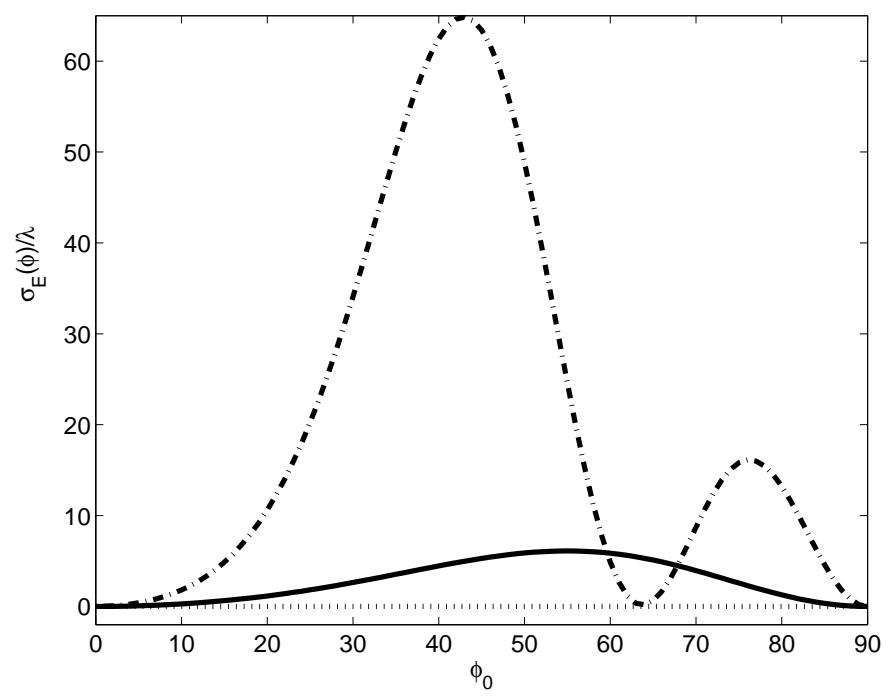

Fig. 4. Bistatic scattering cross section for a plane wave E-polarized incident at an angle $\varphi_{0}=\pi / 4$ when $\zeta=1$. The results shown correspond to $c=1$ (dotted line), $c=\pi$ (solid line), and $c=10$ (dash-dot line).

$$
\begin{array}{r}
\left.H_{2 z}\right|_{\xi=\xi_{1}}=16 \sum_{l=0}^{\infty} \frac{1}{N_{2 \ell}^{(e)}}\left[\tilde{b}_{\ell} \operatorname{Re}_{2 \ell}^{(1)}(\mathrm{c}, \xi)+\right. \\
\left.\left(\operatorname{Re}_{2 \ell}^{(1)}\left(\mathrm{c}, \xi_{0}\right)+\tilde{\mathrm{d}}_{\ell}\right) \operatorname{Re}_{4 \ell}^{(4)}\left(\mathrm{c}, \xi_{1}\right)\right] \operatorname{Se}_{2 \ell}\left(\mathrm{c}, \eta_{0}\right) \operatorname{Se}_{2 \ell}(\mathrm{c}, \eta) .
\end{array}
$$

\section{NUMERICAL RESULTS}

As examples of the computation of the expressions derived in the previous two sections, we provide results for bistatic radar cross sections, currents induced along the metallic walls, and radiated field. The computation of the Mathieu functions was performed using some of the Fortran subroutines provided in [8]. However, these subroutines are based on the Golstein-Ince normalization [9], [10],

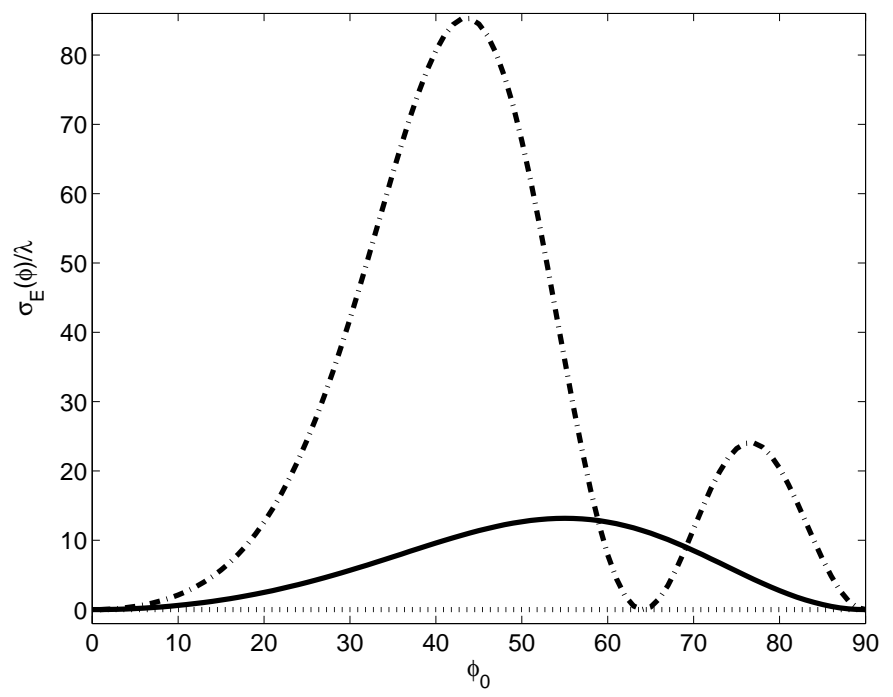

Fig. 5. Bistatic scattering cross section for a plane wave E-polarized incident at an angle $\varphi_{0}=\pi / 4$ when $\zeta=1 / 2$. The results shown correspond to $c=1$ (dotted line), $c=\pi$ (solid line), and $c=10$ (dash-dot line).

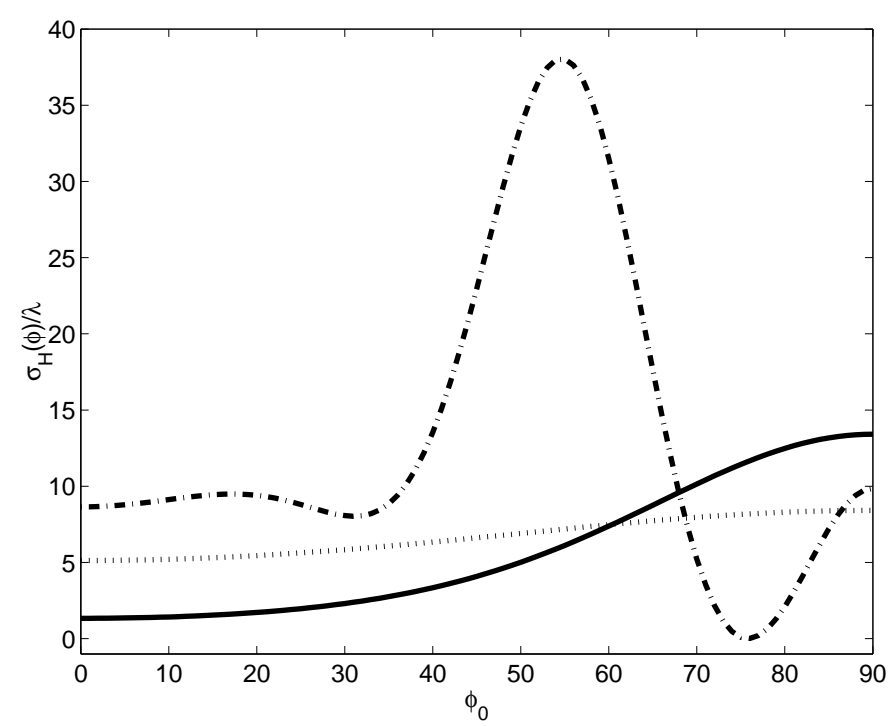

Fig. 6. Bistatic scattering cross section for a plane wave H-polarized incident at an angle $\varphi_{0}=\pi / 4$ when $\zeta=1$. The results shown correspond to $c=1$ (dotted line), $c=\pi$ (solid line), and $c=10$ (dash-dot line).

[11] so they were appropriately modified to account for the normalization used in this work. In particular, these routines were translated to Fortran 90 so that verification of computations at quadruple precision could be performed.

In all the figures that follow, we refer to a cavity with size $\xi_{1}=2$. Results are presented for three values of the dimensionless parameter $c$ given in (8): $1, \pi, 10$ to account for different cases for the size of the aperture $O D$ of Fig. 1 with respect to the wavelength $\lambda$.

The first result, shown in Fig. 4, is the radar cross section (26) for the E-polarized plane wave (11) incident at an angle $\varphi_{0}=\pi / 4$ when $\zeta=1$. When $c=1$ (dotted line) the aperture is smaller than the wavelength and the RCS does not vary as much as it does for the two other cases where the aperture is equal to the wavelength, $c=\pi$ (solidline), or greater than it, $c=10$ (dash-dot line). When the material properties are changed, the shape of the RCS curves remains qualitatively the same but the magnitudes change, as it is shown in Fig. 5 that analyzes the RCS when $\zeta=1 / 2$. The RCS for the H-polarized plane wave (29) incident at an angle $\varphi_{0}=\pi / 4$ when $\zeta=1$ is given in Fig. 6.

The currents induced on the metallic walls of the structure of Fig. 1 are of interest because they provide a way to compare results with other numerical methods such as discussed in [5], [6]. Fig. 7 shows the currents $\left|J_{z}\right|$ induced by the E-polarized plane wave (11), incident at an angle $\varphi_{0}=\pi / 4$ when $\zeta=1$. These results were computed using eqs. (21)-(25) with the help of the Shanks transform acceleration method applied only to the imaginary part of the terms of the series involved, similar to what is described in [12].

Results for the electrical line source (44) located outside the trench at $\left(\xi_{0}=1.5, v_{0}=\pi / 6\right)$ when $\zeta=1$ are given in Fig. 8. Note that there is no singular behavior of $\left|J_{z}\right|$ 

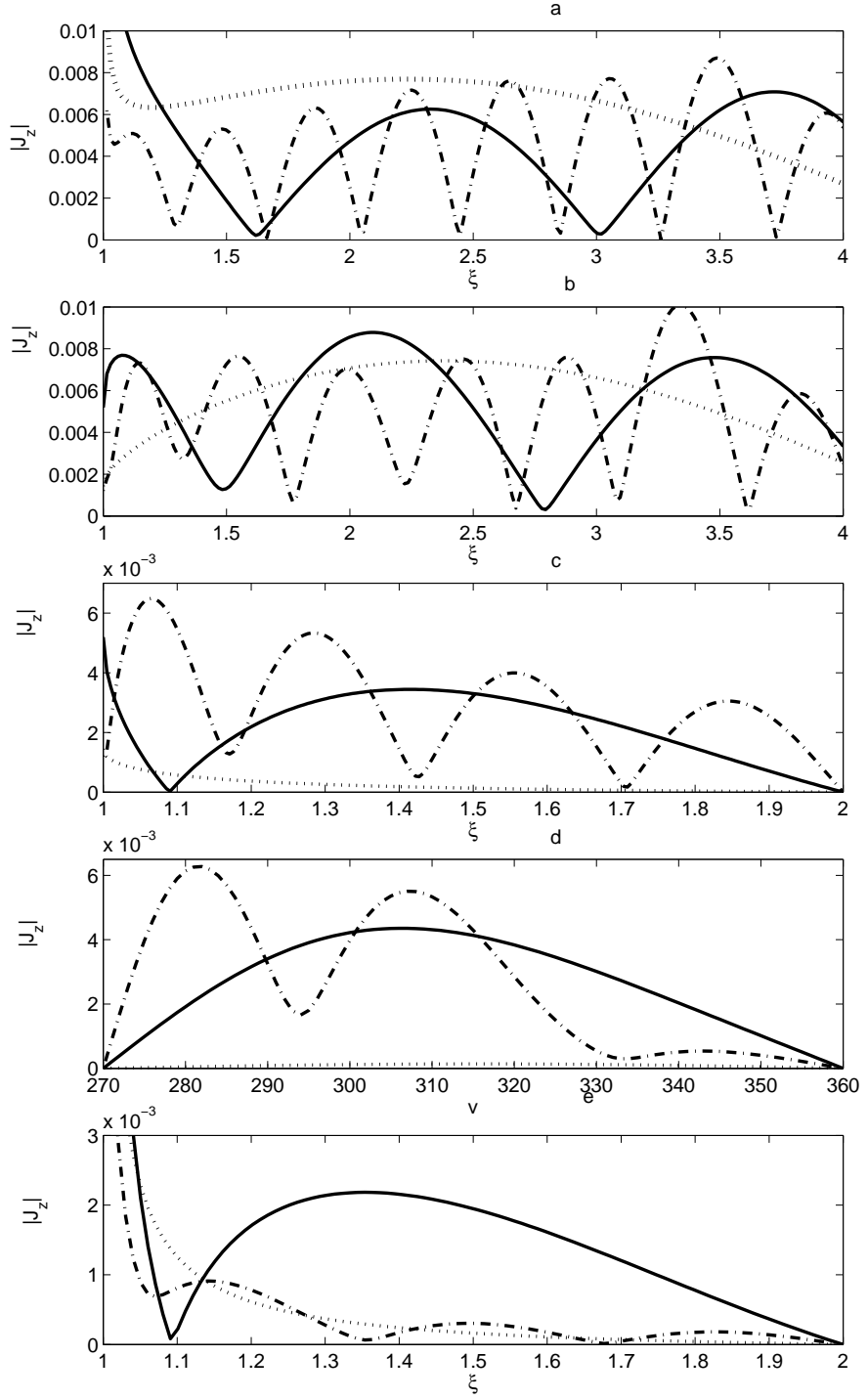

Fig. 7. Currents induced on the metallic walls of the structure of Fig. 1 due to an unit amplitude plane wave electrically polarized and incident at an angle $\varphi_{0}=\pi / 4$. Current $\left|J_{z}\right|$ along $D E$ is shown in (a); current $\left|J_{z}\right|$ along $O A$ is shown in (b); current $\left|J_{z}\right|$ along $O B$ is shown in (c); current $\left|J_{z}\right|$ along $B C$ is shown in (d); and current $\left|J_{z}\right|$ along $D C$ is shown in (e). The results shown in (a-e) correspond to $c=1$ (dotted line), $c=\pi$ (solid line), and $c=10$ (dash-dot line)

in part c) around $\xi=1$. These results were computed using eqs. (54)-(58). In particular, in addition to the use of the aforementioned acceleration technique, the geometrical optics contributions were computed using the direct expression in terms of Hankel functions instead of using its expansion with Mathieu functions.

Results for an electric line located inside the trench at $\left(\xi_{0}=1.5, v_{0}=-5 \pi / 12\right)$ when $\zeta=1 / 2$ are reported in Fig. 9. These results were computed using eqs. (66)-(70). As before, Shanks acceleration technique was used and the geometrical optics terms were evaluated using the direct expressions in terms of Hankel functions.

Fig. 10 gives $\left|J_{z}\right|$ induced by a line source located inside the trench at $\left(\xi_{0}=1.5, v_{0}=-5 \pi / 12\right)$ when $\zeta=2$. These results were computed using eqs. (86)-(90) and were com-
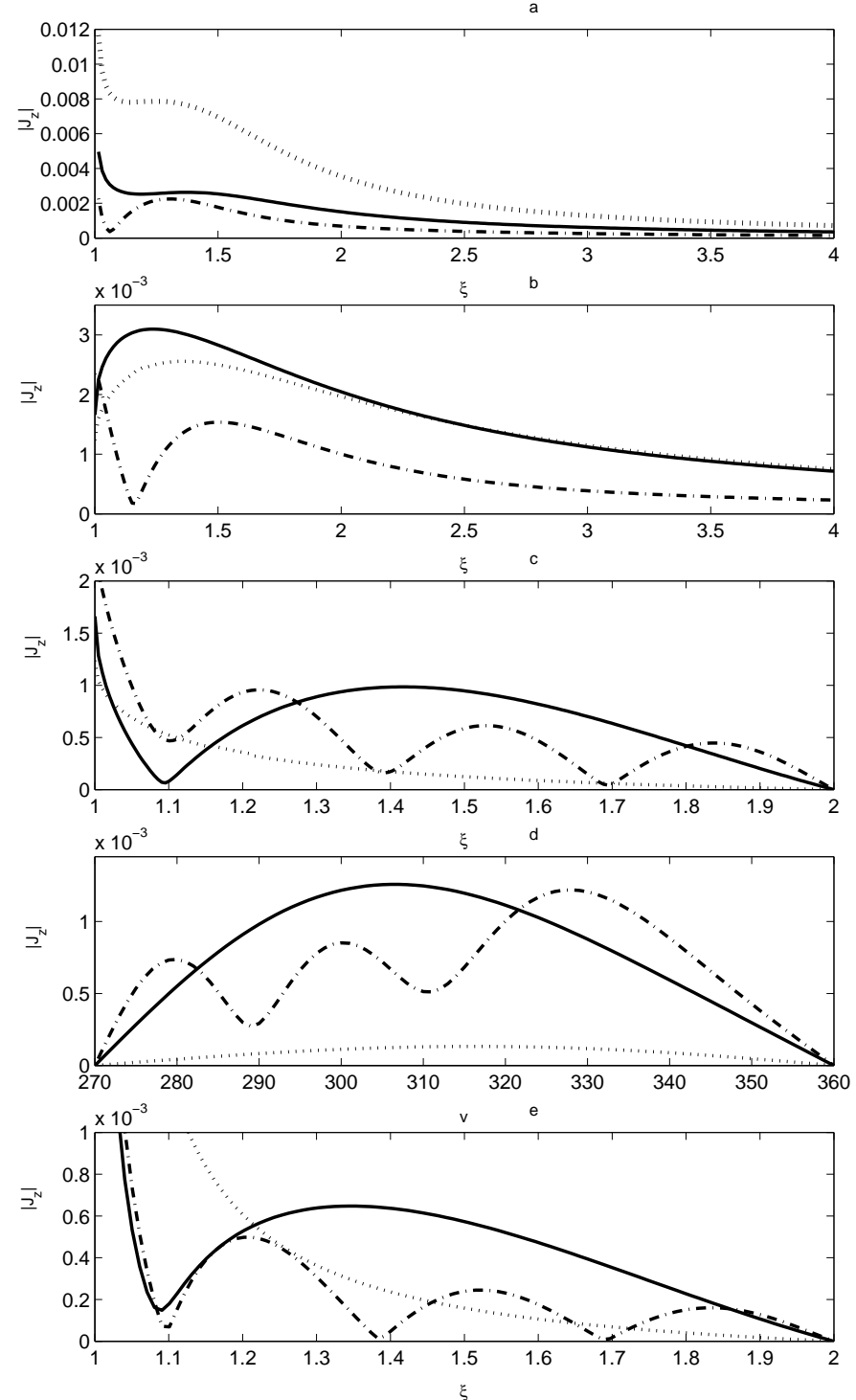

Fig. 8. Currents induced on the metallic walls of the structure of Fig. 1 due to an electric line source located at $\left(\xi_{0}=1.5, v_{0}=\pi / 6\right)$ when $\zeta=1$. Current $\left|J_{z}\right|$ along $D E$ is shown in (a); current $\left|J_{z}\right|$ along $O A$ is shown in (b); current $\left|J_{z}\right|$ along $O B$ is shown in (c); current $\left|J_{z}\right|$ along $B C$ is shown in (d); and current $\left|J_{z}\right|$ along $D C$ is shown in (e). The results shown in (a-e) correspond to $c=1$ (dotted line), $c=\pi$ (solid line), and $c=10$ (dash-dot line).

puted using Shanks acceleration technique as well as with a direct evaluation of the geometrical optics contribution using Hankel functions.

All figures 7-10 have in common that a more complex behavior of $\left|J_{z}\right|$ is observed for larger values of $c$ (dash-dot line, $c=10)$.

Another common feature among all figures 7-10 is the continuity of $\left|J_{z}\right|$ while moving along the metallic surfaces around points $B$ and $C$ of Fig. 1. In fact, since there are no localized currents at such points, the continuity around $B$ is proven by the behavior at $\xi=2$ of part c) and at $v=3 \pi / 2$ of part d), whereas the continuity around $C$ is guaranteed by the behavior around $v=2 \pi$ of part $\mathrm{d}$ ) and $\xi=2$ of part e). 

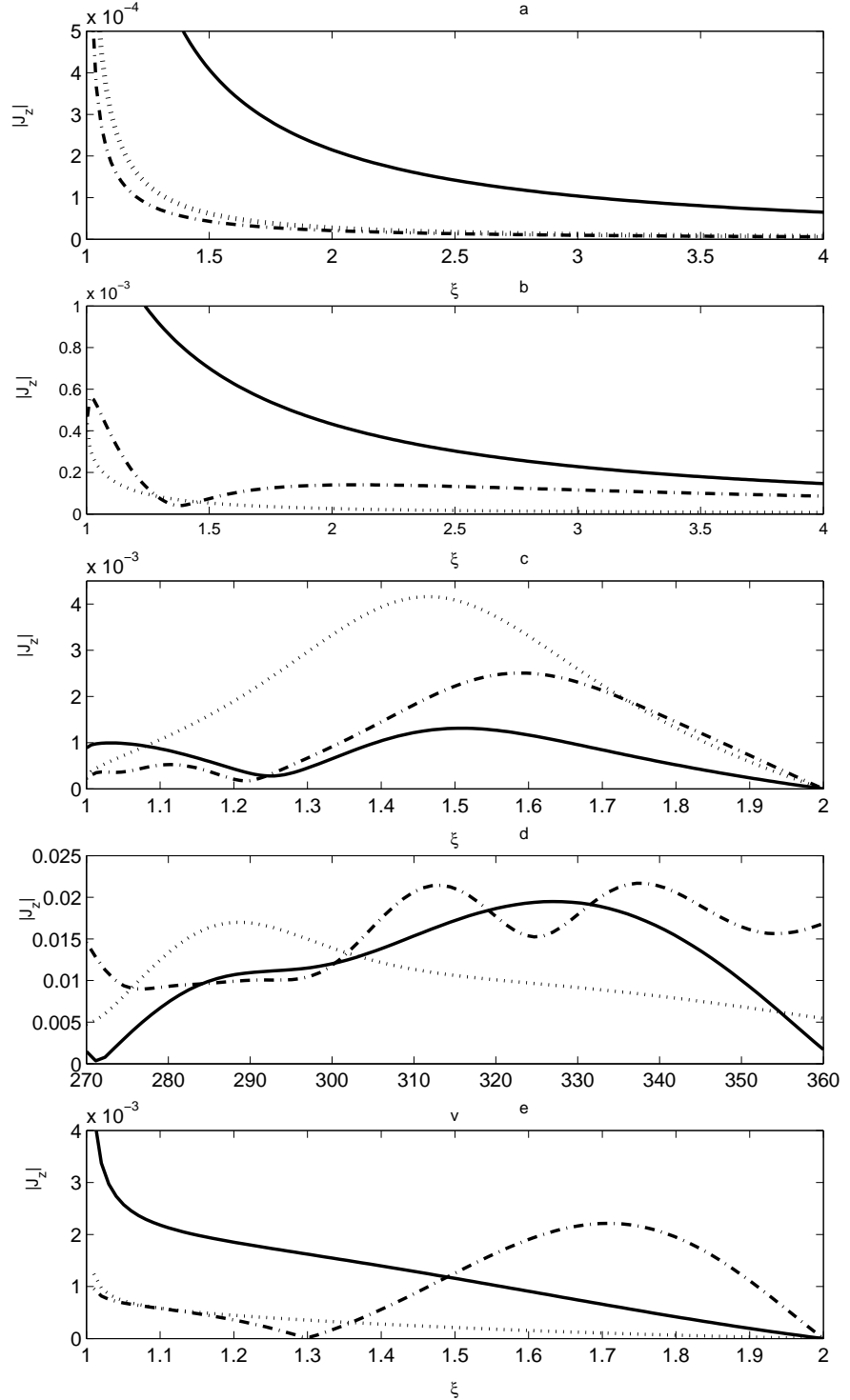

Fig. 9. Currents induced on the metallic walls of the structure of Fig. 1 due to an electric line source located at $\left(\xi_{0}=1.5, v_{0}=-5 \pi / 12\right)$ when $\zeta=1 / 2$. Current $\left|J_{z}\right|$ along $D E$ is shown in (a); current $\left|J_{z}\right|$ along $O A$ is shown in (b); current $\left|J_{z}\right|$ along $O B$ is shown in (c); current $\left|J_{z}\right|$ along $B C$ is shown in (d); and current $\left|J_{z}\right|$ along $D C$ is shown in (e). The results shown in (a-e) correspond to $c=1$ (dotted line), $c=\pi$ (solid line), and $c=10$ (dash-dot line).

All parts a) and e) of the figures related to E-polarized sources, i.e. Fig. 7-9, show singular behaviors at $\xi=1$. These singularities occur near the edge of the baffle $D C$ at point $D$ of Fig. 1 and are due to the factor $1 / \sqrt{\xi^{2}-1}$ that appears in (21) and (22) for plane wave incidence, (54) and (55) for a line source outside the trench, (66) and (67) for a line source inside the trench. Note that this singular behavior is not observed in Fig. 10 that refers to a magnetic line source.

As a last example, Fig. 11 shows the magnitude of the electric field due to an electric line located inside the trench at $\left(\xi_{0}=1.5, v_{0}=-5 \pi / 12\right)$. The magnitude $|E z|$ is evaluated along the hyperbola with $\eta=1 / 2$ when $\zeta=1 / 2$. This geometry may be used to examine the field radiated by a
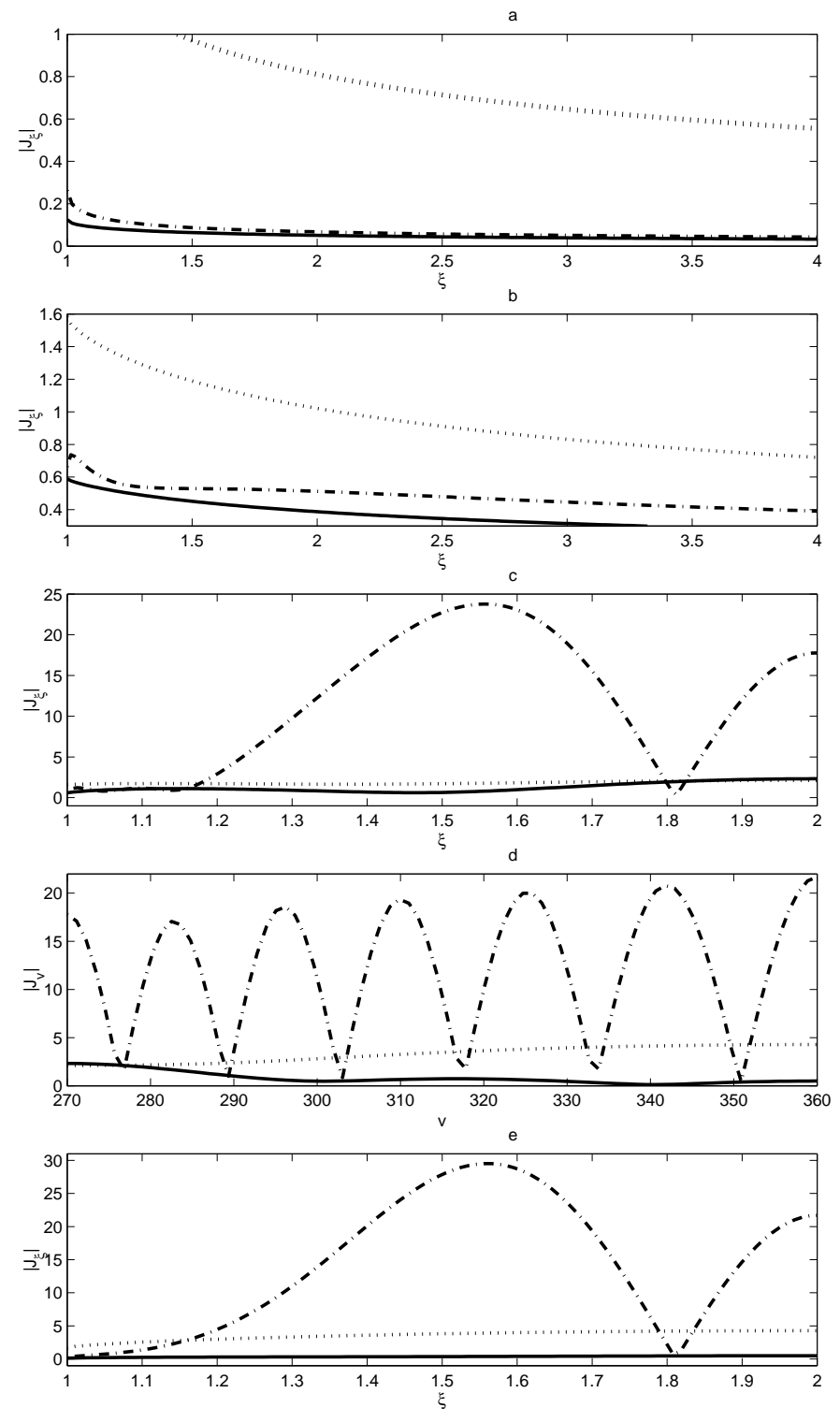

Fig. 10. Currents induced on the metallic walls of the structure of Fig. 1 due to a magnetic line source located at $\left(\xi_{0}=1.5, v_{0}=\right.$ $-5 \pi / 12)$ when $\zeta=2$. Current $\left|J_{\xi}\right|$ along $D E$ is shown in (a); current $\left|J_{\xi}\right|$ along $O A$ is shown in (b); current $\left|J_{\xi}\right|$ along $O B$ is shown in (c); current $\left|J_{v}\right|$ along $B C$ is shown in (d); and current $\left|J_{\xi}\right|$ along $D C$ is shown in (e). The results shown in (a-e) correspond to $c=1$ (dotted line), $c=\pi$ (solid line), and $c=10$ (dash-dot line).

wire that is located inside the trench of Fig. 1. In addition, the field computed for this situation may be compared with the field computed by other methods, similar to what is shown in [4]. As before, Shanks acceleration technique was used and the geometrical optics terms were evaluated using the direct expressions in terms of Hankel functions. The strongest field contribution is observed when $c=\pi$, i.e. $d / \lambda=1$, which may suggest the existence of a resonance condition that causes a better coupling with the field outside the trench. One should also observe the change of slope at $2 y / d=0$, due to the transition from the interior of the cavity to the exterior, as well as to the change of material. 


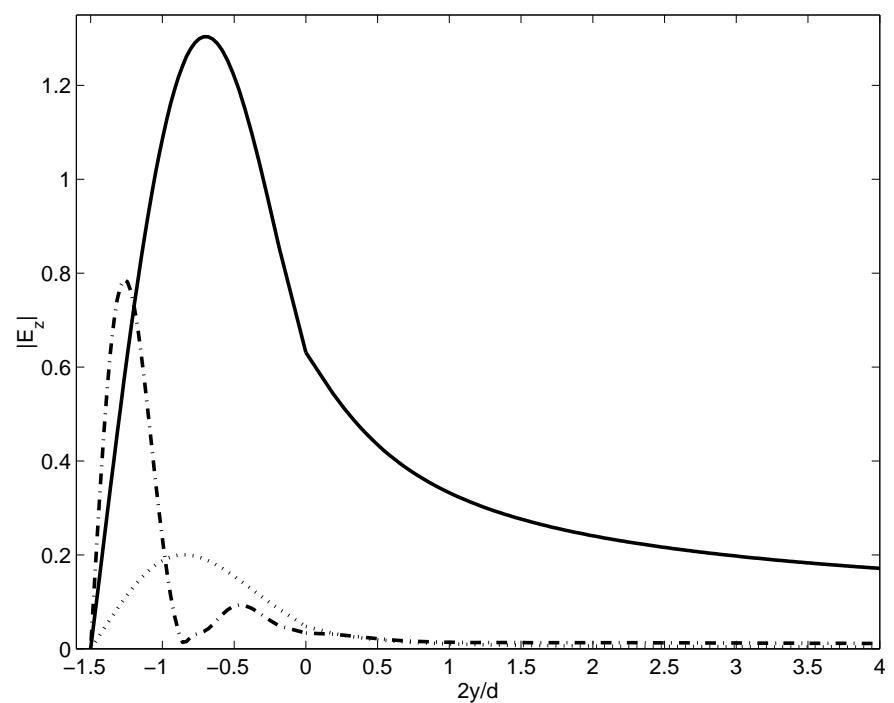

Fig. 11. Magnitude of the electric field along the hyperbola $\eta=1 / 2$ due to an electric line source located at $\left(\xi_{0}=1.5, v_{0}=-5 \pi / 12\right)$ when $\zeta=1 / 2$. The results correspond to $c=1$ (dotted line), $c=\pi$ (solid line), and $c=10$ (dash-dot line).

\section{CONCLUSION}

Exact analytical solutions have been derived for plane wave and line source incidence on a slotted quarterelliptical trench in a corner. These new canonical solutions are important not only per se, but also because they provide benchmarks for the validation of frequency-domain codes involving geometries with trenches, sharp edges, and different penetrable materials.

Numerical results based on these exact solutions have been obtained and discussed. It would be interesting to investigate the approximate forms that these exact solutions take in the low-frequency $(c \ll 1)$ and high-frequency $(c \gg 1)$ limits. However, these studies would require a detailed knowledge of the behavior of the Mathieu functions in the Stratton-Chu notation for small and large values of the parameter $c$, and will constitute the topic of future investigations.

\section{REFERENCES}

[1] J. A. Stratton, Electromagnetic Theory, McGraw-Hill, New York, 1941

[2] J. J. Bowman, T. B. A. Senior, and P. L. E. Uslenghi, Electromagnetic and Acoustic Scattering by Simple Shapes, Hemisphere Publishing Corporation, New York, 1987.

[3] P.L.E. Uslenghi, "Exact penetration, radiation and scattering for a slotted semielliptical channel filled with isorefractive material," IEEE Trans. Antennas Propagat., vol. 52, no. 6, pp. 1473-1480, June 2004.

[4] D. Erricolo, M. D. Lockard, C. M. Butler, and P. L. E. Uslenghi, "Numerical analysis of penetration, radiation, and scattering for a 2D slotted semielliptical channel filled with isorefractive material," PIER, vol. 53, pp. 69-89, 2005.

[5] D. Erricolo, M.D. Lockard, C.M. Butler, and P.L.E. Uslenghi, "Comparison among currents on surfaces inside and near a semielliptical channel filled with isorefractive material that backs a slotted plane: currents computed by analytical formulas and by integral equation methods," in Proc. Intl. Conf. on Electromagnetics in Advanced Applications (ICEAA'O3), Torino, Italy, Sept 2003, pp. 469-474.

[6] D. Erricolo, M.D. Lockard, C.M. Butler, and P.L.E. Uslenghi, "Currents on conducting surfaces of a semielliptical-channel- backed slotted screen in an isorefractive environment," IEEE Trans. Antennas Propagat., 2003, accepted.

[7] P.L.E. Uslenghi, "Exact electromagnetic penetration througha gap in a corner," in Proc. 1999 IEEE-APS/URSI Intl. Symp, Orlando, FL, USA, July 1999, p. 164.

[8] S. Zhang and J.-M. Jin, Computation of Special Functions, Wiley, New York, 1996.

[9] S. Goldstein, "Mathieu functions," Camb. Phil. Soc. Trans. vol. 23, pp. 303-336, 1927

[10] E. L. Ince, "Tables of elliptic cylinder functions," Roy. Soc. Edin. Proc., vol. 52, pp. 355-423, 1932.

[11] M. Abramovitz and I. A. Stegun, Handbook of Mathematical Functions, Dover Publications, Inc, New York, 1970.

[12] D. Erricolo, "Acceleration of the convergence of series containing Mathieu functions using Shanks transformation," IEEE Antennas and Wireless Propagation Letters, vol. 2, pp. 58-61, 2003.

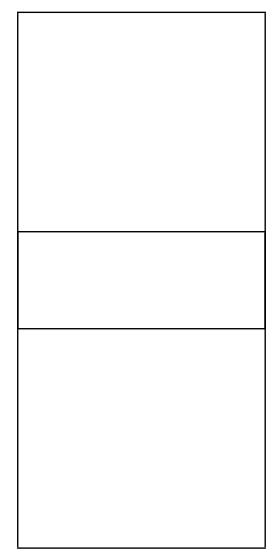

Danilo Erricolo (S'97 - M'99 - SM'03) For a biography and photo, see the September 2004 issue of this TRANSACTIONS.

Piergiorgio L. E. Uslenghi (SM '70 - F '90) For a biography and photo, see the June 2004 issue of this TRANSACTIONS. 